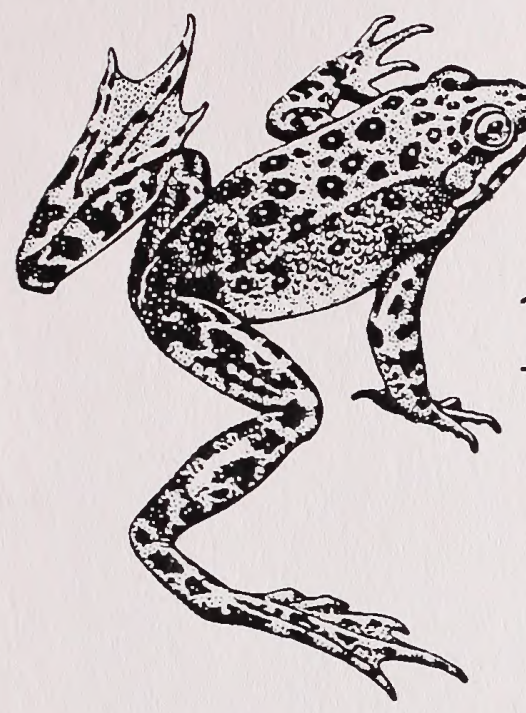

\title{
1996 Survey for Columbia Spotted Frogs in the Owyhee Mountains of Southwestern Idaho
}

QL

84.2

.2352

no.

$97-13$ by

James C. Munger

Aaron Ames

Bruce Barnett 



\section{Survey for Columbia Spotted Frogs in the Owyhee Mountains of Southwestern Idaho}

A challenge cost-share agreement between

U.S. Bureau of Land Management and

Boise State University

June 18, 1997

James C. Munger

Aaron Ames

Bruce Barnett

Department of Biology

Boise State University

Boise, ID 83725

\section{INTRODUCTION}

The Habitat Conservation Assessment and Strategy for the Columbia Spotted Frog in Idaho recently identified the highest priority research needs for this species as identifying the status of and threats to the Owyhee Mountains population of this species (Munger et al, 1995b). We simply do not know whether this population is stable or is suffering declines. Similarly, it is unknown how strongly various land use practices, such as grazing, affect spotted frogs in arid environments. Addressing these questions will determine the need for management of spotted frogs in SW Idaho.

The USFWS (Tumer 1993) elevated to C1 status 3 of the 4 populations of the Columbia spotted frog (Rana luteiventris), based on documented extinctions and declines as well as the existence of threats due to loss of habitat by conversion to pastures and dewatering of wetlands to supply irrigation needs, impact of livestock grazing, development, restricted habitat availability, and introduction of predaceous fish and amphibians. The USFWS has subsequently modified its classification system: all C2 species were dropped, and most C1 species became "candidate" species. One such candidate population, the Great Basin population of the Columbia spotted frog, occupies isolated mountain ranges in southwestern Idaho, southeastern Oregon, and Nevada. Its elevation to candidate status was based on loss of populations in a number of areas of 
Nevada. Although technically a part of the Great Basin population, the isolated population occupying the Owyhee Mountains of SW Idaho is relatively poorly known. Our knowledge of this population is based primarily on two previous BLM-financed surveys (Munger et al. 1994, 1995a) that identified eleven populations and gathered habitat data. We were able to identify several trends: spotted frogs tended to be near permanent, slow-moving water with little vegetation, and were found more often then expected in National Wetland Inventory's (NWI) palustrine system, shrubscrub class, and seasonally-flooded regime. Evidence of recent grazing was found to have a measurable but modest negative association with spotted frogs.

The present study represents the third year of survey. As we go to press with this report, a fourth year of surveying is underway, using many of the same methods as the present study. For that reason, we will in this report give only a preliminary analysis of habitat data. Following the completion of the Summer 1997 survey, we plan to produce a comprehensive report that will give a more in-depth analysis of distribution, habitat affinities, and land-use effects.

\section{MATERIALS AND METHODS}

Spotted Frog Occurrence: We continued to survey as much of the remaining wetland area in the Owyhee Mountains as possible. We examined topographic maps and land use maps and chose to survey those stretches of stream that seemed, based on their low gradient, likely to harbor spotted frogs. Streams and other wetlands were walked in a slow and deliberate manner, and all likely places in which adult frogs might reside were examined. All still waters are examined and/or dipnetted to ascertain the presence of tadpoles. UTM's for all sites where frogs were found were taken using a Geoexplorer GPS unit. We typically took a minimum of 120 points with a PDOP of 6 or less. Locations were later corrected in the laboratory using base station data from the $\mathrm{McCall}$, the Idaho City, and the Shoshone base stations. All frogs were sexed, weighted, measured (snout to vent), and a toe was collected (and stored in 95\% ethanol) for later age and genetic analysis.

Habitat associations: To expand our data base on habitat associations, we took habitat measures at sites where adult or larval spotted frogs were found and at randomly chosen sites. For the area within $5 \mathrm{~m}$ of the frog sighting, we measured the width and depth of the water body; measured the air and water temperature; classified it as an oxbow, sidebow, pond, pool, riffle, or run; classified the bank slope as shallow, moderate, or steep; estimated the percent cover or willow, sagebrush, grass, forb, duff, rock, bare, algae, and other aquatic vegetation; gave the site a 0 to 3 rating for overall grazing impact; and counted the number of cow pats within $5 \mathrm{~m}$.. On a larger scale (on the order of a $50 \mathrm{~m}$ segment), we classified the water body as a stream, pond, stock pond, or reservoir; classified the gradient as still, slow, moderate, fast, or cascade; measured the bank height on both sides of the water; classified the valley type as V, trough, flat-bottom, or box canyon; classified the sinuousness as low, moderate, high, or braided; and scored the presence or absence of downcutting, dead willows, and historic braids or oxbows.

Population Monitoring: At Stoneman Creek we continued our yearly census of the frogs. See 

Munger 1995 for a description of the exact stretch of stream surveyed. On May 24, 1996, workers moved through the area, capturing and freeze-branding all frogs encountered. On May 31,1996 , the crew returned and captured all frogs encountered, scoring them as to whether they were marked or not. A simple Lincoln Index was used to estimate the population size. At Rock Creek, we captured and freeze-branded all frogs encountered on May 15 and 16, 1997, along the ca. 2 mile stretch of federal land in Sections 23, 25, 26, and 35 of T8S R2W. We returned to recapture frogs on May 23, 1997. Again, a Lincoln index was used to estimate population size.

Incidental Captures: We noted all encounters of other species of reptiles and amphibians. UTM coordinates and elevation were approximated using a single uncorrected GPS reading.

\section{RESULTS}

\section{Spotted Frog Occurrence}

We found adult or subadult Columbia spotted frogs at the following water bodies: Little Blue Creek, Cottonwood Creek, tributary to Pleasant Valley Creek, Duck Creek, Johnston Reservoir, Camel Creek, Pole Creek, Rail Creek Reservoir, and Old Man Creek (Appendix I). We found eggs or larvae at the following water bodies: Camel Creek, Cottonwood Creek, Duck Creek, Little Blue Creek (Appendix I). This substantially increases the number of breeding sites known for the Owyhees, bringing the total to 11 sites.

\section{Habitat Associations}

The association of the presence of spotted frogs to categorical variables were analyzed using contingency tables (Table 1). For non-categorical variables, we compared spotted frog sites to non-frog sites using a Multivariate Analysis of Variance (Table 2).

Of note are the following:

1. Spotted Frogs tended to be found more often then expected in or around ponds or pools and less often than expected in or near riffles or runs (Table 1).

2. Spotted frog sites had a warmer water temperature than non-frog sites (Table 2).

3. Spotted frog sites were lower in sagebrush, rock, and forbs, but higher in grass and algae than nonfrog sites (Table 2).

4. Frog sites were of lower gradient, had lower banks, and had banks of lower slope than nonfrog sites (Table 2, Figure 1).

5. Spotted frog sites had less evidence of recent grazing than non-frog sites: a lower overall rating and a lower number of cowpats (Tables 1 and 2; Figures 1 and 2).

6. There is evidence that long-term degradation of the habitat negatively affects spotted frogs: spotted frog sites had lower bank heights and greater sinuousness than non-frog sites. In addition, spotted frog sites tended to be found more often than expected where no downcutting or lateral erosion were present (Tables 1 and 2; Figures 1 and 2).

7. We found old braids present at 79 sites and dead willows at 17 sites, indicating that in some areas the water table previously had been higher and the stream course previously had 

been more sinuous. Spotted frogs were found more often than expected in sites with dead willows, indicating that frogs tend to prefer willowed sites (which have perennial water) even if the water table has dropped (Table 1).

\section{Population Monitoring}

Numbers of spotted frogs at Stoneman Creek have remained relatively stable for the last three years, with on the order of 100-150 frogs (Figure 3). We estimate that a population of about 80 frogs occupies Rock Creek. At this time there is no indication of decline in the number of frogs at Stoneman Creek.

\section{Incidental Captures}

We captured two additional amphibian species, the Pacific treefrog (Pseudacris regilla) and the western toad (Bufo boreas) (Appendices I and II). We also captured individuals of eleven reptile species: the rubber boa (Charina bottae), the racer (Coluber constrictor), the western whiptail (Cnemidophorus tigris), the western rattlesnake (Crotalus viridis), the leopard lizard (Gambelia wislizenii), the gopher snake (Pituophis catenifer), the shorthorned lizard (Phrynosoma douglassii), the desert horned lizard (Phrynosoma platyrhinos), the sagebrush lizard (Sceloporus graciosus), the western fence lizard (Sceloporus occidentalis), and the western terrestrial garter snake (Thamnophis elegans) (Appendix II).

\section{DISCUSSION}

\section{$\underline{\text { Habitat }}$}

Habitat measures taken in 1996 indicate that Spotted frogs are most likely to be found in areas with low gradient, shallow banks, and with pools and/or oxbows. While we have been somewhat successful at finding such habitat on federal lands, much of this prime habitat occurs on private land. This is because areas with low gradient (which typically occur in gentle valleys) were the areas that were originally chosen by homesteaders. It is also interesting to note that in a number of cases, frogs we found on federal land were located immediately adjacent to private land. It is clear, therefore, that the cooperation of private landowners could substantially increase the potential for protecting appropriate habitat for spotted frogs.

\section{Grazing}

We found evidence that spotted frogs were found more often in areas with less evidence of recent grazing: spotted frog sites were given (on average) lower ratings of grazing evidence and had fewer cow pats. However, except for preventing the sort of overgrazing that leads to long-term loss of habitat (see next paragraph), the institution of new management actions concerning grazing should await further study specifically designed to examine the effects of grazing. Such a study is scheduled to begin in the summer of 1998. There are several reasons for awaiting the results of a more intensive study:

1. The focus of the present study was not to gather information on grazing, but was focussed on the discovery of new populations. Because the focus was not on grazing, information on grazing 

was gathered in a relatively informal way. An intensive study will have a more rigorous study design and a reduced chance for bias and other potential sampling problems.

2. Although trends were apparent for grazing rating and for cow pat number, the trends were only modest. Examination of Figure 2A shows that frogs were found in areas of relatively high grazing intensity.

3. Severely overgrown areas may be relatively poor habitat for spotted frogs.

It is our opinion that the long-term effects of overgrazing are a greater problem than short-term effects of grazing. Long-term overgrazing can lead to compaction of soil and loss of stream side vegetation, which in turn lead to increased erosion and downcutting. Downcutting leads to a lowering of the water table and a less sinuous water course. The net result is a loss of the oxbows and pools that are important components of the habitat of spotted frogs. For although frogs may occasionally feed along runs and riffles, feeding frogs tend to be concentrated along pools and in oxbows, and breeding is restricted to oxbows, ponds, and occasionally occurs in very slow moving pools. The prevention of downcutting and the restoration of downcut areas should be high priority management goals.

\section{LITERATURE CITED:}

Munger, J., L. Heberger, D. Logan, W. Peterson, L. Mealy, and M. Cauglin. 1994. A survey of the herpetofauna of the Bruneau Resource Area, with focus on the spotted frog, Rana pretiosa. Idaho Bureau of Land Management Technical Bulletin.

Munger, J., M. Gerber, M. Cauglin, and T. Bert. 1995a. Status and habitat associations of the spotted frog, Rana pretiosa, in southwestern Idaho. Idaho Bureau of Land Management Technical Bulletin.

Munger, J., C. Peterson, M. McDonald, and T. Carrigan. 1995b. Habitat Conservation Assessment and Strategy for the Spotted Frog. Prepared for Idaho Department of Fish and Game.

Turner, J. F. 1993. Endangered and threatened wildlife and plants; finding on petition to list the Spotted Frog. Federal Register 58(87):27260-27263 

Figure 1. Selected habitat measures.

A. Slope of bank. B. Gradient of water body. C. Sinuosity of the water body. D. Height of the bank.
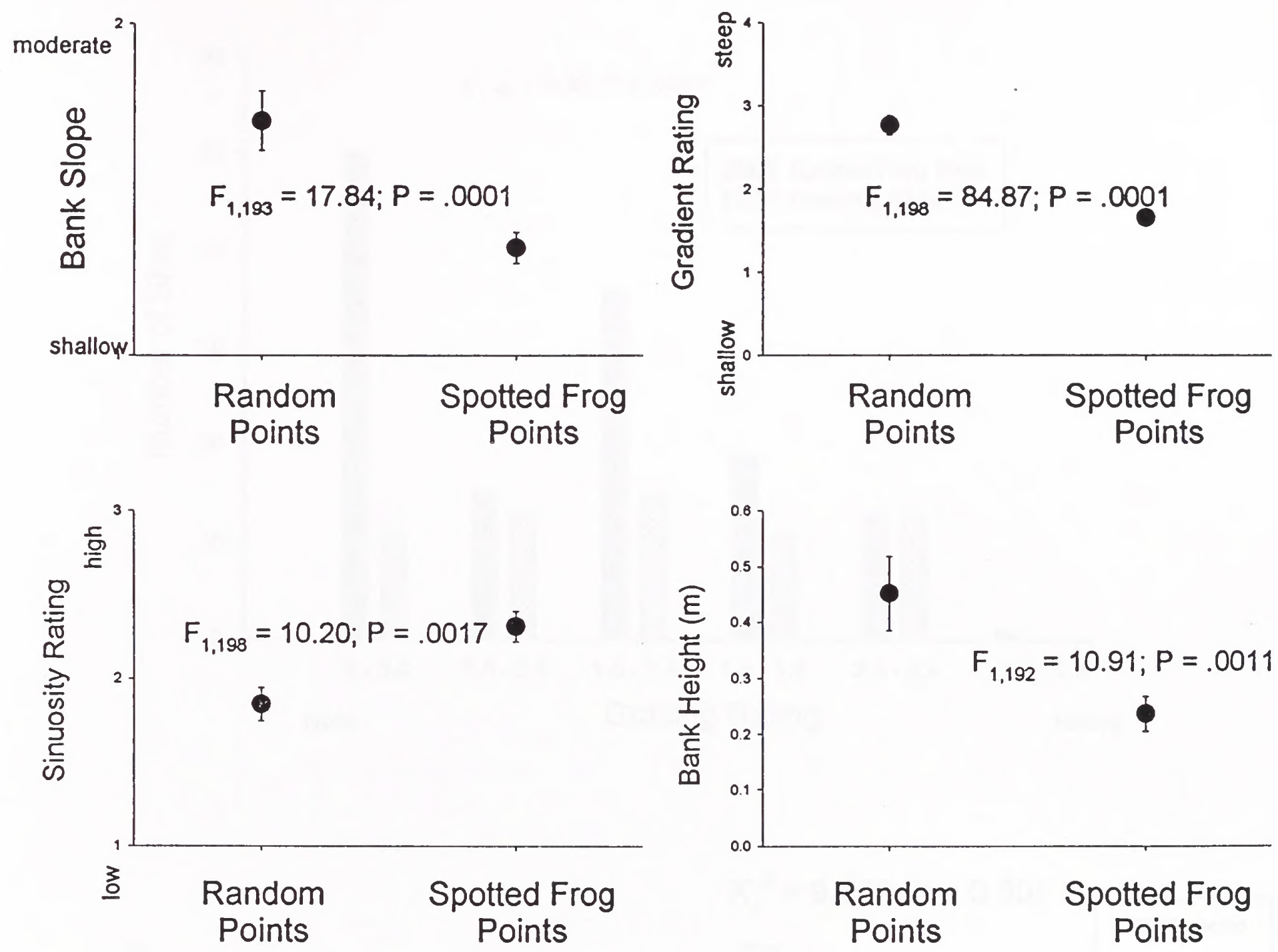

Figure 2. Grazing-related variables. A. Overall grazlng rating $(0-3)$. B. Number of cow pats within $5 \mathrm{~m}$. C. Presence of downcutting.
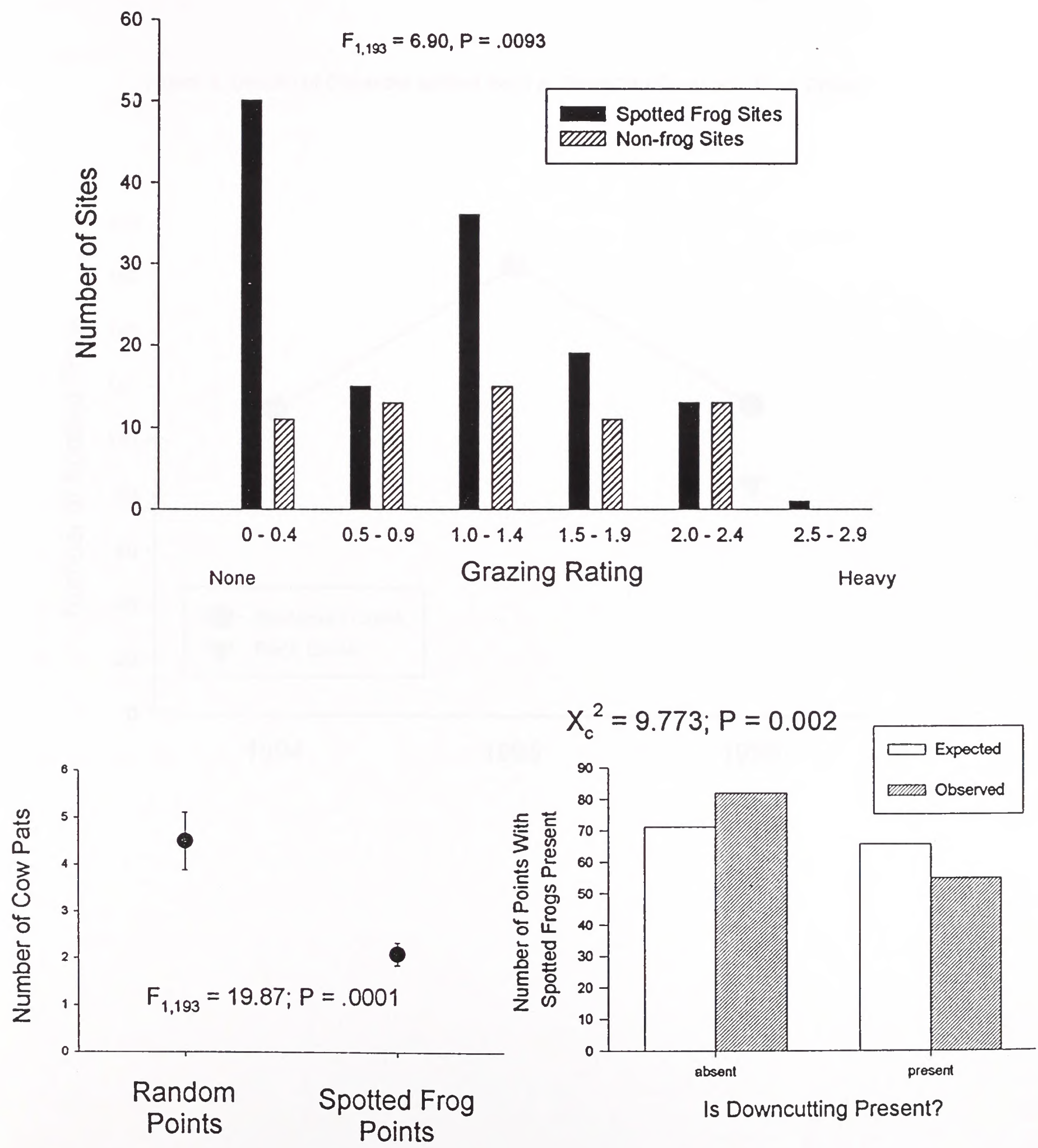


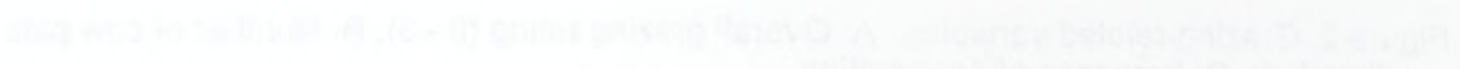


Figure 3. Density of Columbia spotted frogs at Stoneman Creek and Rock Creek.

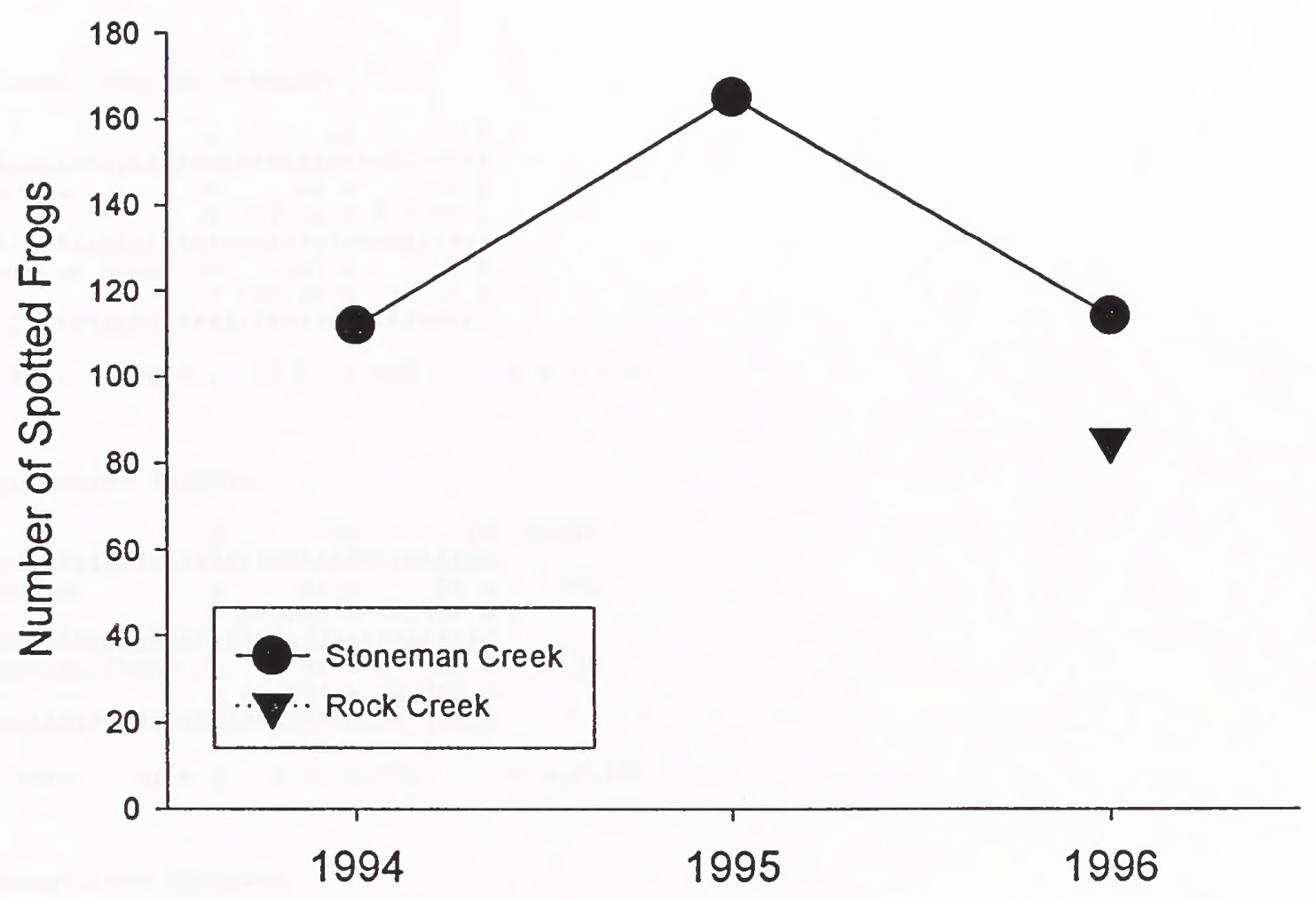



Table 1. Analysis of categorical variables. $0=$ not present, $1=$ present.

DOWNCUTTING PRESENT?

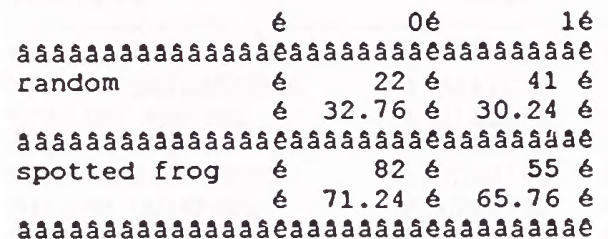

G-test, $\quad d f=1 \quad G=10.856 \quad P=0.001$

LATERAL EROSION PRESENT?

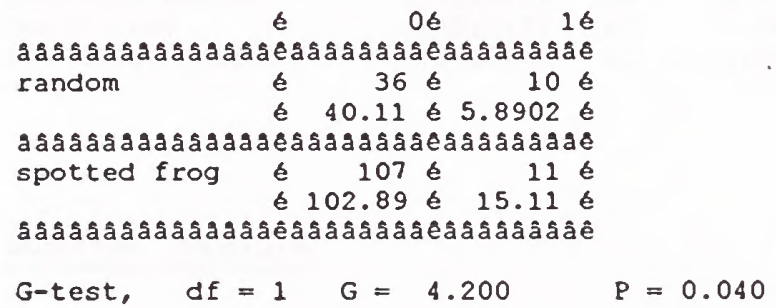

OLD BRAIDS PRESENT?

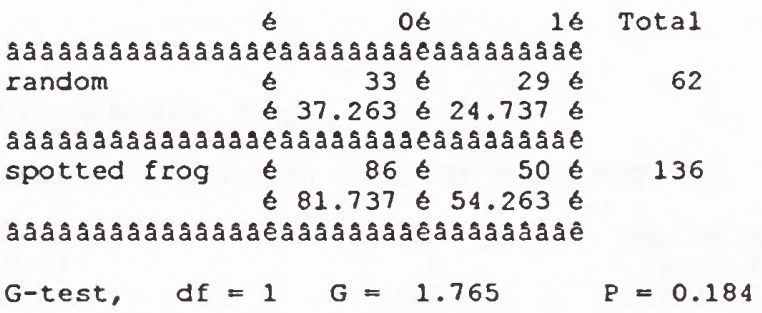

DEADWILLOWS PRESENT?

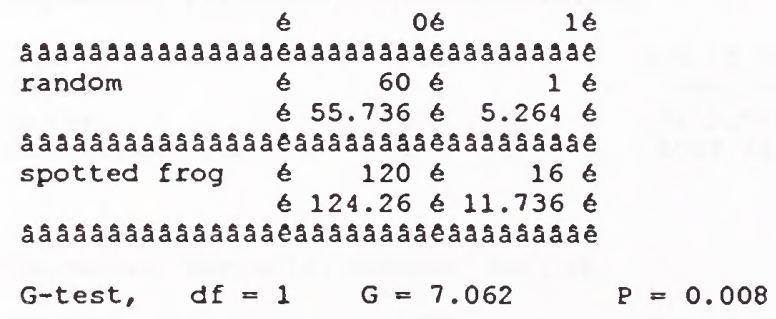

WATER BODY TYPE

éoxbow éoxpool épond épool épool/runériffle érlffrun érun ésldebow ésidepondé

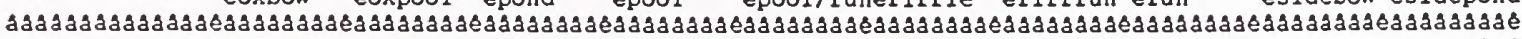

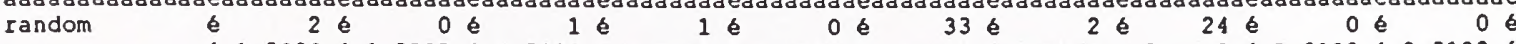
e 1.5909 é 1.2727 é 8.5909 é 7.9545 e 0.3182 e 14.318 é 0.6364 é 20.682 é 7.3182 é 0.3182 é

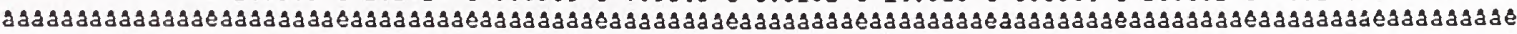

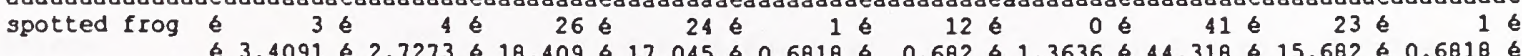

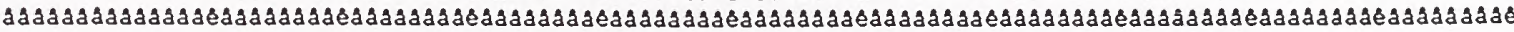

G-test, $\quad d f=9 \quad G=86.210 \quad P=0.001$ 

Table 2. Analysis of meristic varlables. A conservative evaluation of the statistical significance of P-values would be to compare them to a Bonferron1 adjusted $\alpha=.0032$.

Means for Means for Random Sites

\begin{tabular}{lrrr} 
VaIIable & Mean & N & Std Error \\
\hline WIDTH OE WATER BODY & 2.4674603 & 63 & 0.7803036 \\
WATER TEMPERATURE & 14.3141026 & 39 & 0.6416939 \\
GRAZING RATING & 1.0452381 & 63 & 0.0848307 \\
OE COW PATS & 4.4920635 & 63 & 0.6109066 \\
BANK DEGREDATION & 1.7063492 & 63 & 0.0888244 \\
WILLOW COVERAGE & 18.1269841 & 63 & 3.1171439 \\
SAGEBRUSH COVERAGE & 7.3968254 & 63 & 1.0390579 \\
GRASS COVERAGE & 44.8412698 & 63 & 3.1759470 \\
FORB COVERAGE & 12.1428571 & 63 & 1.4944610 \\
DUEE COVERAGE & 4.0317460 & 63 & 0.9238736 \\
ROCK COVERAGE & 11.1904762 & 63 & 1.9382237 \\
BARE COVERAGE & 8.5873016 & 63 & 1.2807678 \\
ALGAE RATING & 9.0634921 & 63 & 1.9903599 \\
GRADIENT RATING & 2.7698413 & 63 & 0.1196435 \\
BANKHEIGHT & 0.4513492 & 63 & 0.0673527 \\
SINUOSITY RATING & 1.8467742 & 62 & 0.0972851
\end{tabular}

Means for Spotted Erog Sites

\begin{tabular}{lllll} 
Mean & $N$ & Std Error & $\begin{array}{c}\text { ANOVA } \\
\text { P-value }\end{array}$ & $R^{2}$ \\
\hline 12.7811111 & 135 & 2.2328920 & 0.0021 & 0.047036 \\
19.0733333 & 135 & 0.3936129 & 0.0001 & 0.167231 \\
0.7678030 & 132 & 0.0607159 & 0.0093 & 0.034503 \\
2.0809160 & 131 & 0.2347901 & 0.0001 & 0.093544 \\
1.3244275 & 131 & 0.0459530 & 0.0001 & 0.085021 \\
19.9562044 & 137 & 2.2881203 & 0.6465 & 0.001065 \\
3.5401460 & 137 & 0.6657459 & 0.0016 & 0.048935 \\
54.5766423 & 137 & 2.2064380 & 0.0134 & 0.030479 \\
5.1240876 & 137 & 0.6835996 & 0.0001 & 0.108708 \\
1.6277372 & 137 & 0.3274685 & 0.0027 & 0.044685 \\
4.1094891 & 137 & 1.1265312 & 0.0010 & 0.053450 \\
5.0948905 & 137 & 0.7710745 & 0.0155 & 0.029224 \\
28.1021898 & 137 & 2.4397701 & 0.0001 & 0.110141 \\
1.6605839 & 137 & 0.0603976 & 0.0001 & 0.300035 \\
0.2370229 & 131 & 0.0312743 & 0.0011 & 0.053789 \\
2.3070175 & 114 & 0.0920932 & 0.0017 & 0.055398
\end{tabular}

\section{ANOVA TABLES}

Overall Manova

Wilks' Lambda $=0.53436509 \quad E_{16,110}=6.4264 \quad P=0.0001$

\section{Univariate Tests}

Dependent Var1able: WIDTH OF WATER BODY

$\begin{array}{lrr}\text { Source } & \text { DF } & \text { Sum of Squares } \\ \text { EROG } & 1 & 4569.13482251 \\ \text { Error } & 196 & 92571.50512698 \\ \text { Corrected Total } & 197 & 97140.63994949\end{array}$

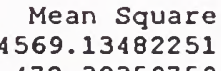

472.30359759
E Value 9.67

$\operatorname{Pr}>E$ 0.0021

Dependent Varlable: WATER TEMPERATURE

$\begin{array}{lrr}\text { Source } & \text { DE } & \text { Sum of Squares } \\ \text { EROG } & 1 & 685.36615584 \\ \text { Error } & 172 & 3412.94874359 \\ \text { Corrected Total } & 173 & 4098.31489943\end{array}$

Mean Square 685.36615584 19.84272525
E Value 34.54

$\mathrm{Pr}>\mathrm{E}$ 0.0001

Dependent Variable: GRAZING RATING

$\begin{array}{lrr}\text { Source } & \text { DE } & \text { Sum of Squares } \\ \text { EROG } & 1 & 3.28248364 \\ \text { Error } & 193 & 91.85423431 \\ \text { Corrected Total } & 194 & 95.13671795\end{array}$

Dependent Varlable: NUMBER OE COW PATS

$\begin{array}{lrr}\text { Source } & \text { DE } & \text { Sum of Squares } \\ \text { EROG } & 1 & 247.31910086 \\ \text { Error } & 192 & 2396.54832182 \\ \text { Corrected Total } & 193 & 2643.86742268\end{array}$

Mean Square 247.31910086 12.48202251
E Value $\quad \operatorname{Pr}>E$ $19.81 \quad 0.0001$

$$
\begin{array}{rrr}
\text { Mean Square } & \text { Evalue } & \operatorname{Pr}>\text { F } \\
3.28248364 & 6.90 & 0.0093 \\
0.47592868 & &
\end{array}
$$



Dependent Variable: BANK DEGRADATION DUE TO GRAZING

$\begin{array}{lrr}\text { Source } & \text { DE } & \text { Sum of Squares } \\ \text { FROG } & 1 & 6.20524370 \\ \text { Error } & 192 & 66.77929238 \\ \text { Corrected Total } & 193 & 72.98453608\end{array}$

Mean Square

6.20524370

0.34780881

E Value

17.84

Pr $>E$

72.98453608

Dependent Variable: WILLOW COVERAGE

$\begin{array}{lrr}\text { Source } & \text { DE } & \text { Sum of Squares } \\ \text { EROG } & 1 & 144.39864674 \\ \text { Error } & 198 & 135500.72135326\end{array}$

Corrected Total $199 \quad 135645.12000000$

Dependent Variable: SAGEBRUSH COVERAGE

$\begin{array}{lrr}\text { Source } & \text { DE } & \text { Sum of Squares } \\ \text { EROG } & 1 & 641.88643784 \\ \text { Error } & 198 & 12475.10856216 \\ \text { Corrected Total } & 199 & 13116.99500000\end{array}$

Dependent Variable: GRASS COVERAGE

$\begin{array}{lrr}\text { Source } & \text { DE } & \text { Sum of Squares } \\ \text { FROG } & 1 & 4090.12204611 \\ \text { Error } & 198 & 130105.85795389 \\ \text { Corrected Total } & 199 & 134195.98000000\end{array}$

Dependent Variable: FORB COVERAGE

$\begin{array}{lrr}\text { Source } & \text { DE } & \text { Sum of Squares } \\ \text { EROG } & 1 & 2125.95020334 \\ \text { Error } & 198 & 17430.60479666 \\ \text { Corrected Total } & 199 & 19556.55500000\end{array}$

Dependent Variable: DUFE COVERAGE

$\begin{array}{lrr}\text { Source } & \text { DE } & \text { Sum of Squares } \\ \text { FROG } & 1 & 249.40389352 \\ \text { Error } & 198 & 5331.95110648 \\ \text { Corrected Total } & 199 & 5581.35500000\end{array}$

Dependent Variable: ROCK COVERAGE

$\begin{array}{lrr}\text { Source } & \text { DE } & \text { Sum of Squares } \\ \text { FROG } & 1 & 2163.80805005 \\ \text { Error } & 198 & 38319.07194995 \\ \text { Corrected Total } & 199 & 40482.88000000\end{array}$

Dependent Variable: BARE COVERAGE

$\begin{array}{lrr}\text { Source } & \text { DE } & \text { Sum of Squares } \\ \text { FROG } & 1 & 526.35873537 \\ \text { Error } & 198 & 17485.03626463 \\ \text { Corrected Total } & 199 & 18011.39500000\end{array}$

Mean Square 684.34707754

Mean Square 641.88643784 63.00559880

Mean Square 4090.12204611 657.10029270

Mean Square 2125.95020334 88.03335756

E Value 24.15

$\operatorname{Pr}>\mathrm{E}$ 0.0001

Mean Square 249.40389352 26.92904599

E Value 9.26

$\mathrm{Pr}>\mathrm{E}$ 0.0027

Mean Square 2163.80805005 193.53066641

E Value $\operatorname{Pr}>\mathrm{E}$ $11.18 \quad 0.0010$

Mean Square 526.35873537 88.30826396
E Value 5. 96 Pr $>$ E 0.0155 144.39864674 

Dependent Variable: ALGAE RATING

$\begin{array}{lrr}\text { Source } & \text { DF } & \text { St.m of Squares } \\ \text { FROG } & 1 & 15642.47962519 \\ \text { Error } & 198 & 126380.31537481 \\ \text { Corrected Total } & 199 & 142022.79500000\end{array}$

15642.47962519

638.28442108

Sum of squares

53.10014830

123.87985170

176.98000000

Error

Corrected Total

198

199

Dependent Varlable: BANK HEIGHT

$\begin{array}{lr}\text { Source } & \text { DF } \\ \text { FROG } & 1 \\ \text { Error } & 192 \\ \text { Corrected Total } & 193\end{array}$

Dependent Variable: SINUOSITY

$\begin{array}{lr}\text { Source } & \text { DF } \\ \text { FROG } & 1 \\ \text { Error } & 174 \\ \text { Corrected Total } & 175\end{array}$

Sum of squares

1.95416428

34.37579925

36.32996353

Sum of squares

8.50665692

145.04874080

153.55539773
Mean Square

F Value

24.51

0.0001

Mean Square

53.10014830

0.62565582

E Value

84.87

$\operatorname{Pr}>\mathrm{F}$

0.0001
E Value 10.91

1.95416428

0.17904062

Mean Square
8.50665692

0.83361345
E Value 10.20

$\mathrm{Pr}>\mathrm{E}$ 0.0017 



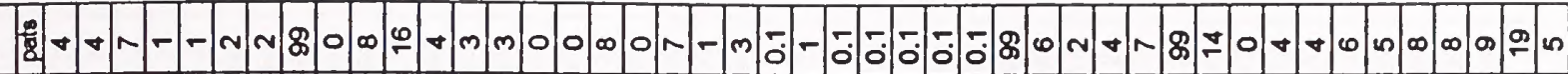
这-

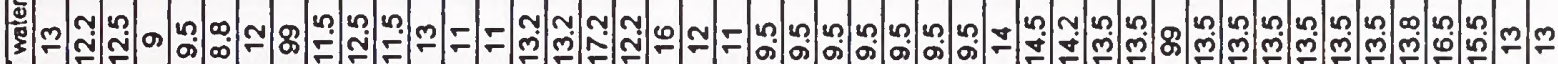

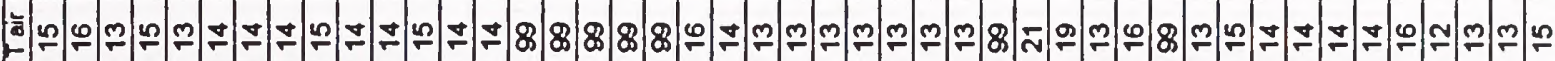

5 - 0 - 1 - -

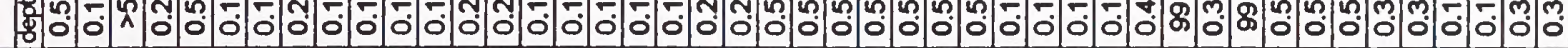

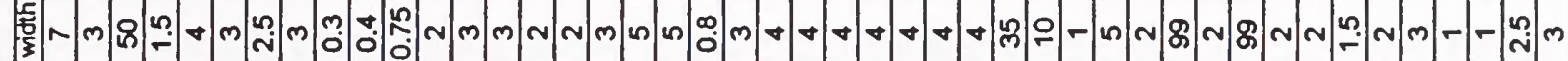

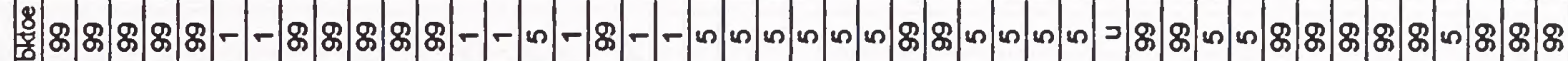

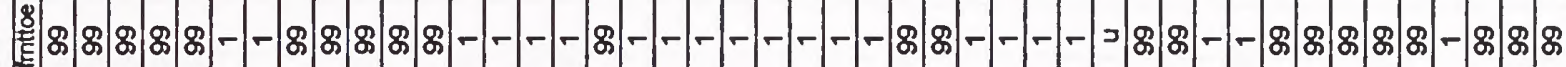
$\$ \& \delta \& \&$ E

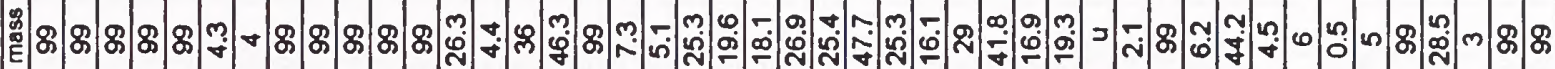

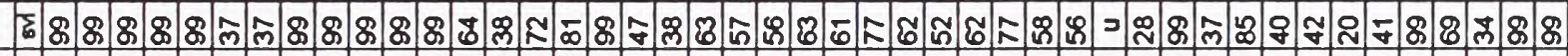

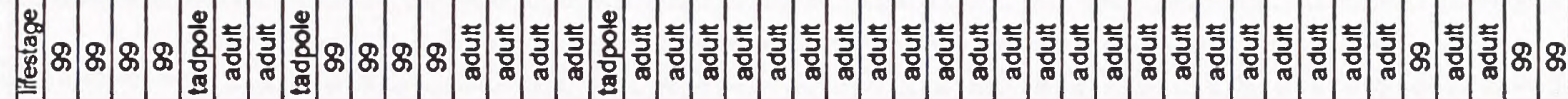

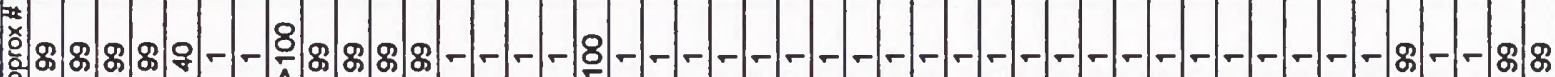
ㅁำ

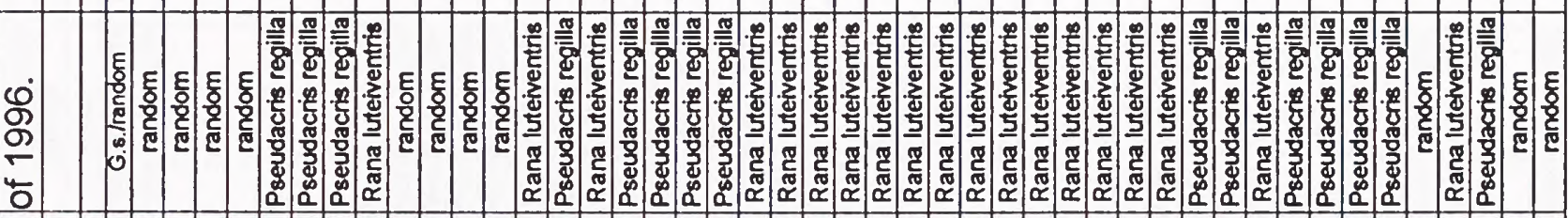
产 亏

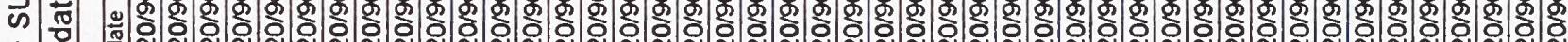

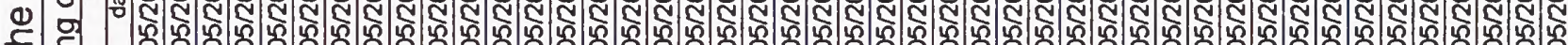

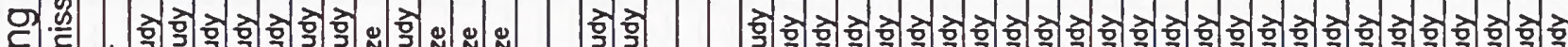
든 음

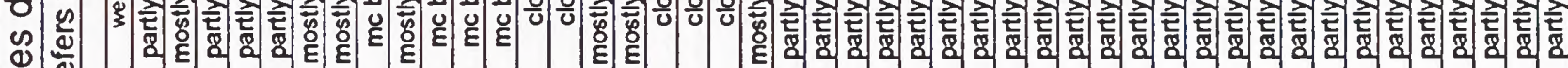

$\frac{\mathscr{Q}}{\mathrm{O}}$

ำำ 至

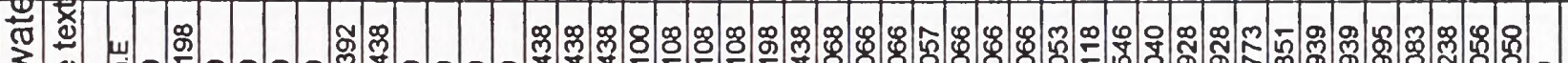

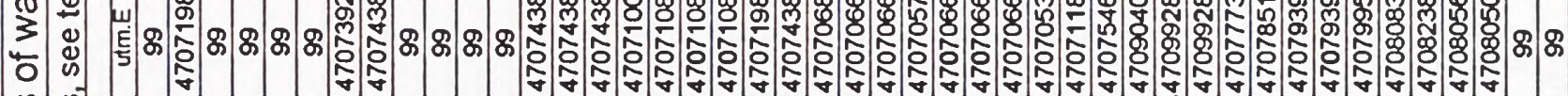

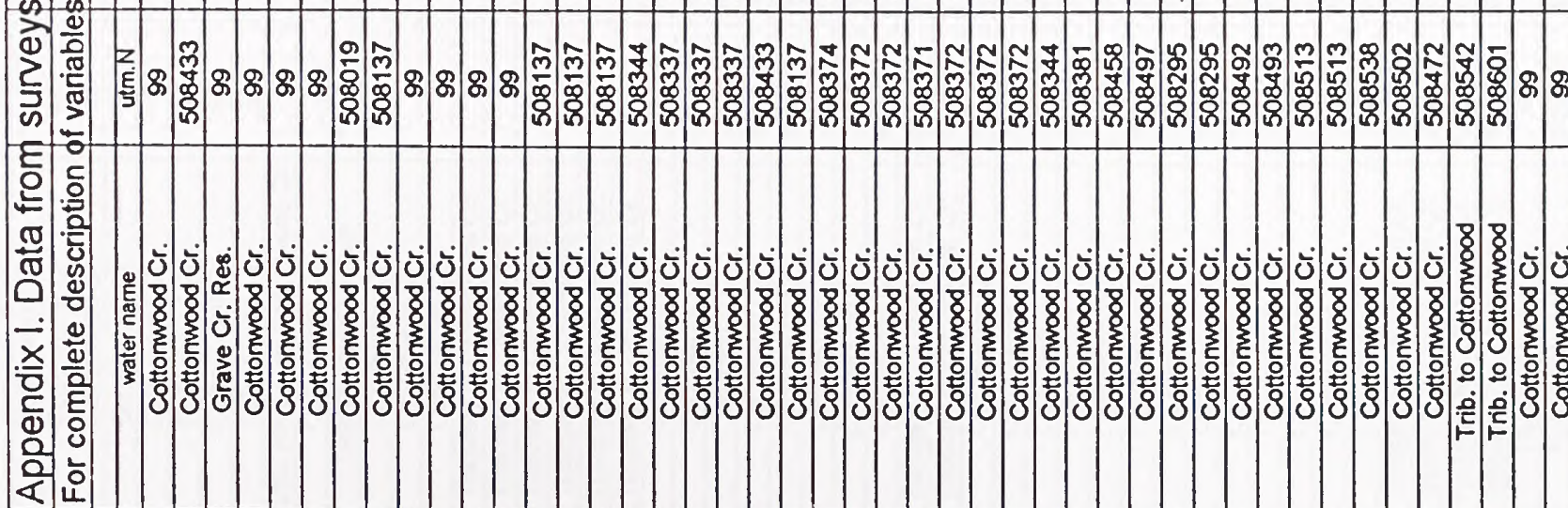



我 으 $\begin{aligned} & n=0 \\ & 0\end{aligned}$ 듣요 ก : ஊே-

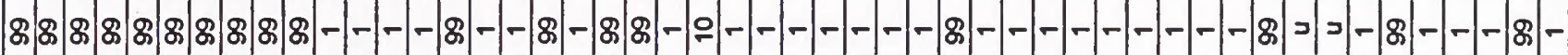

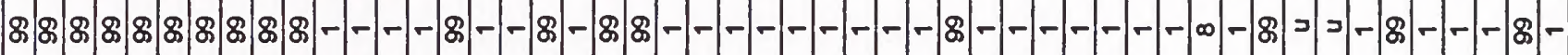

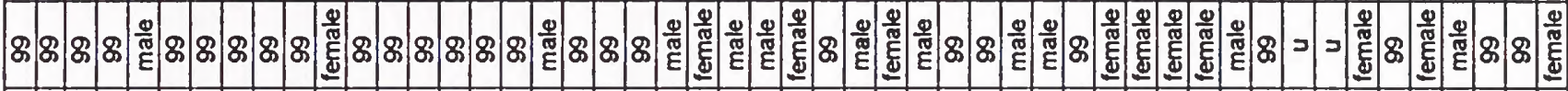

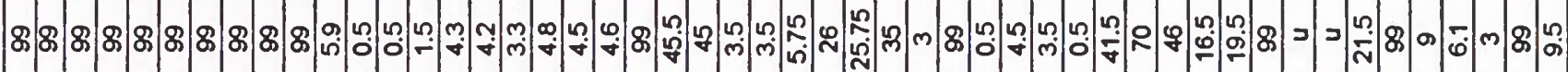

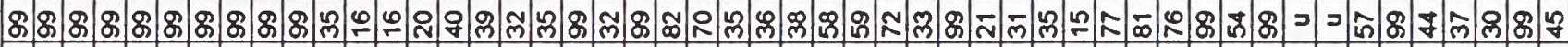

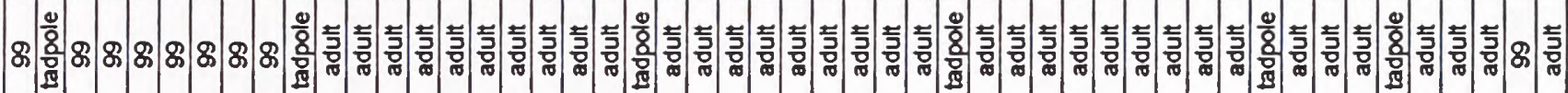

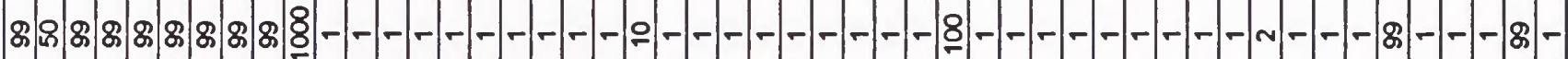

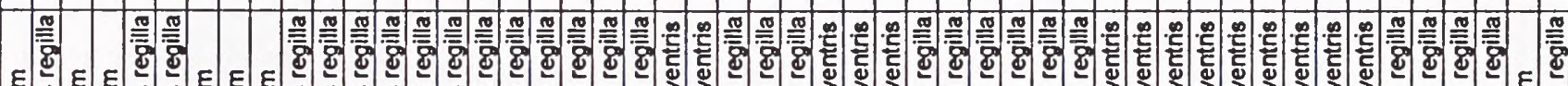

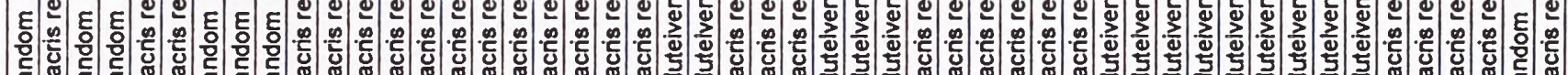

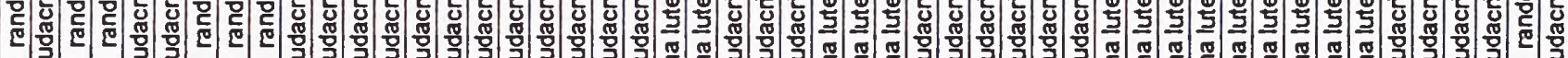

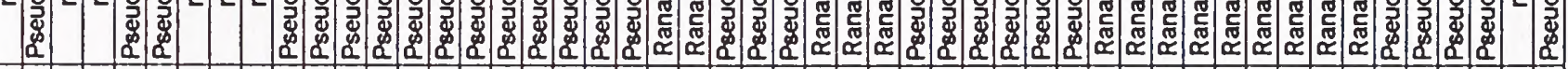
8.8 8

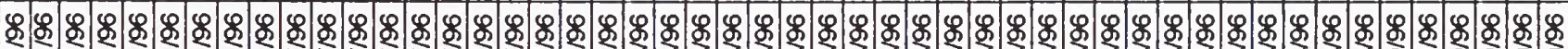

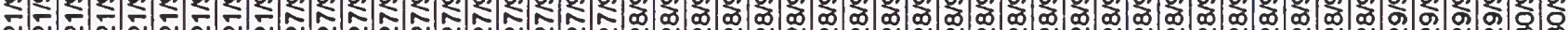

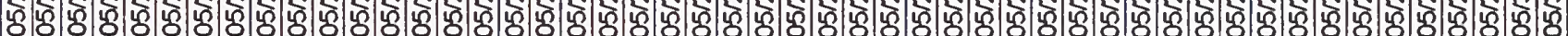

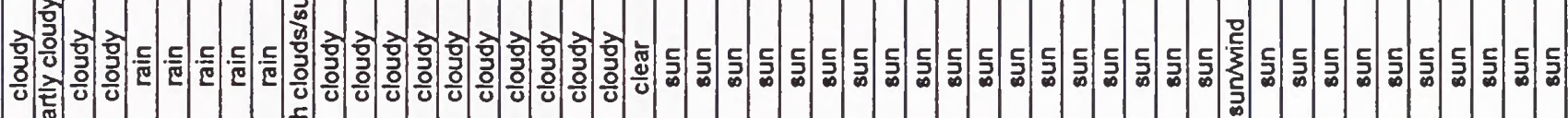

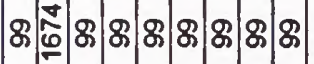
28: ஓ

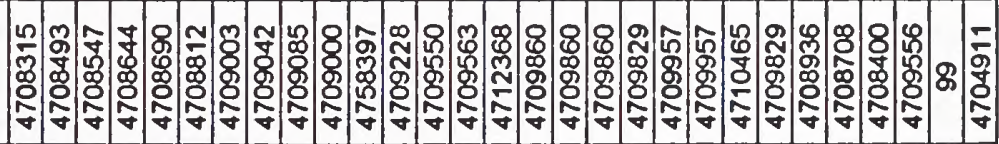

৪

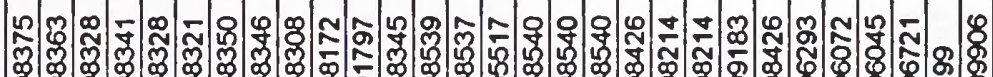

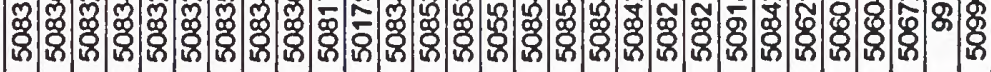

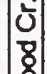

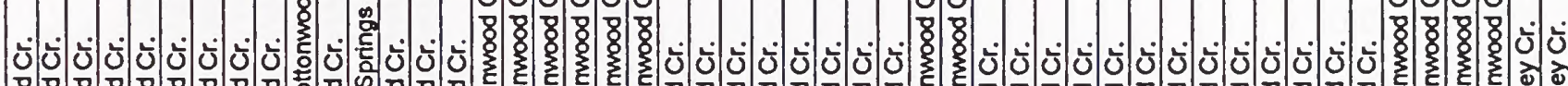

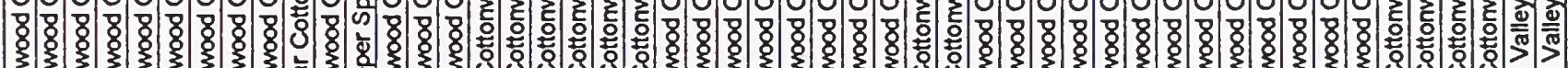

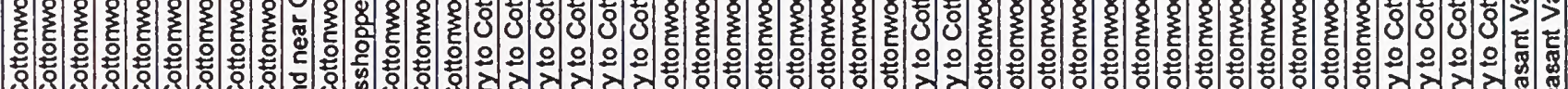
ব) 



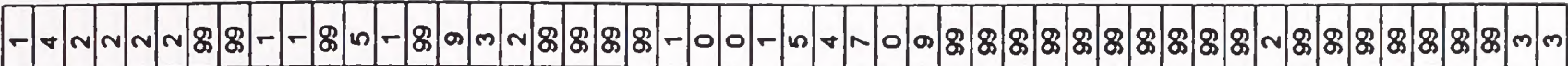
ㄱ-

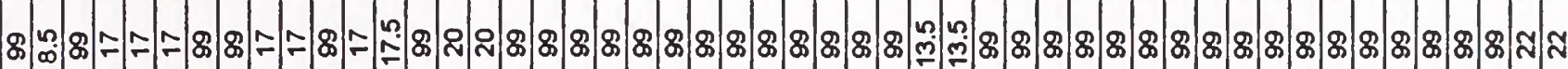

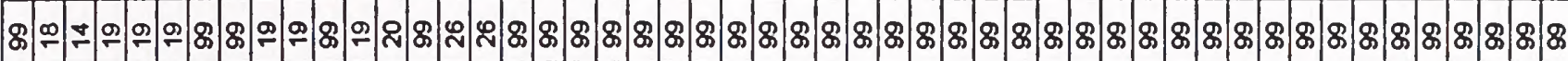
○.

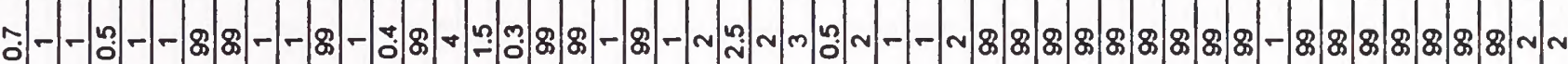

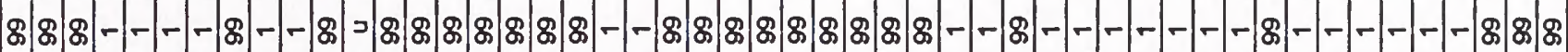

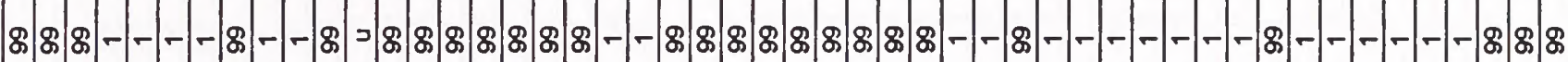

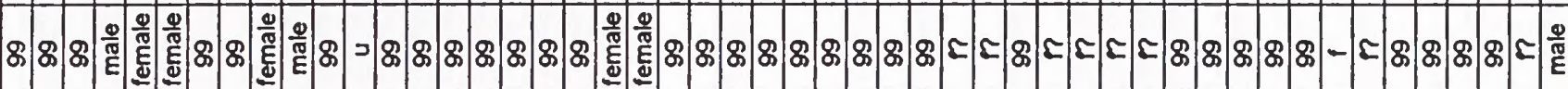

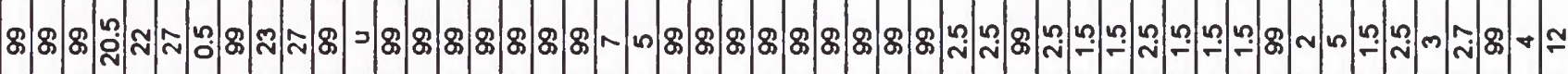

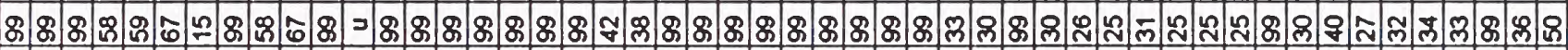

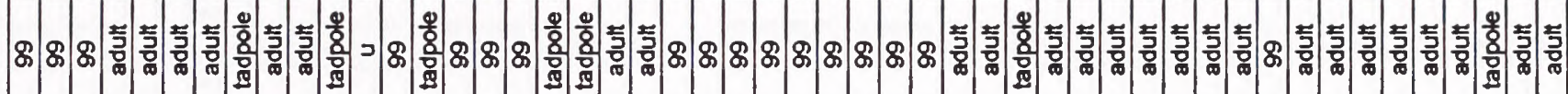

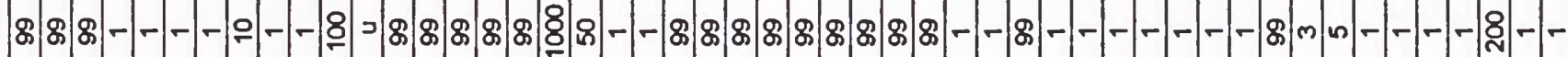

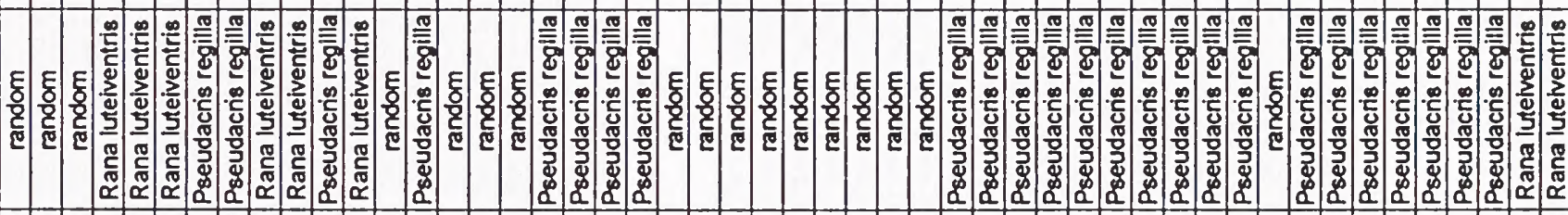

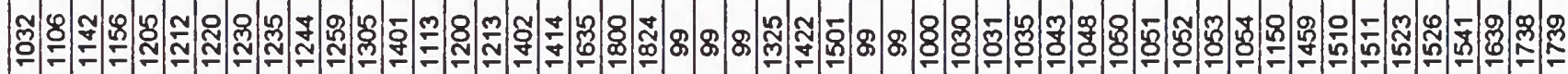

\$.

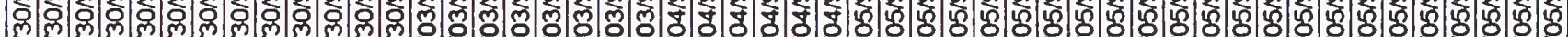

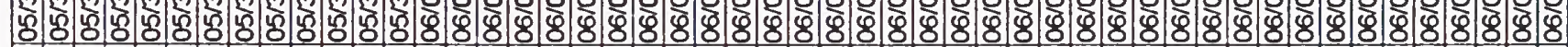

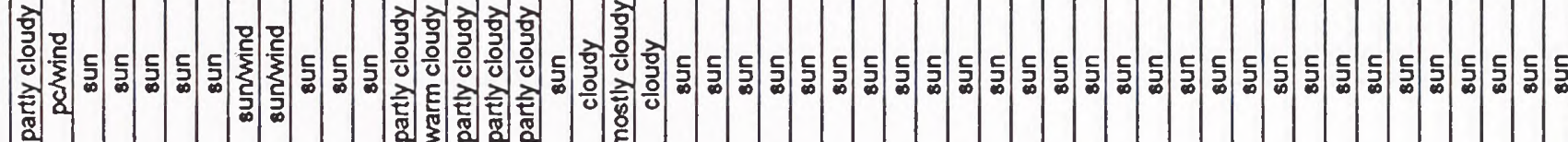

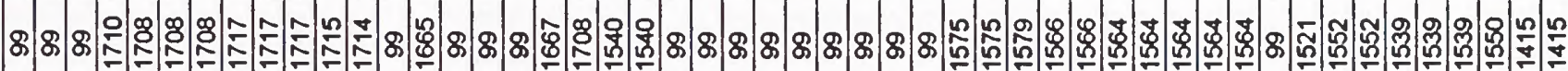
牙

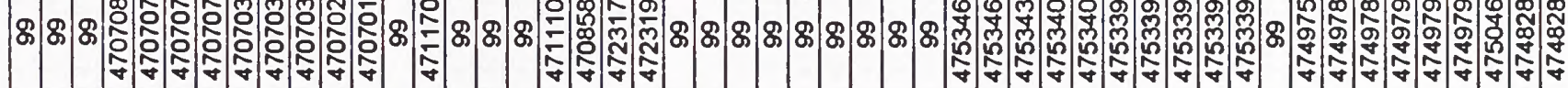

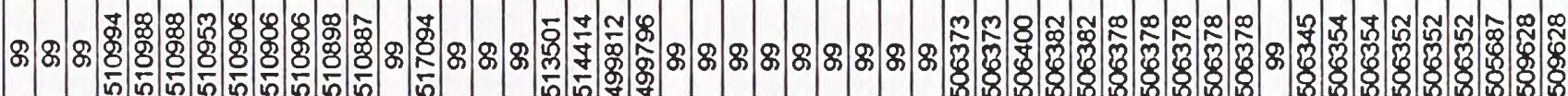

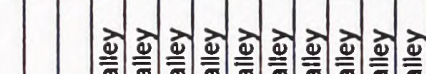

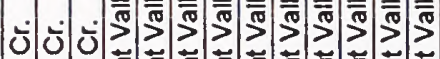

可

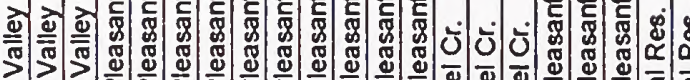

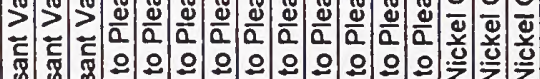

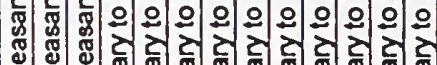

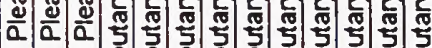

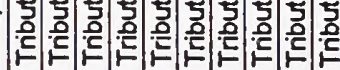

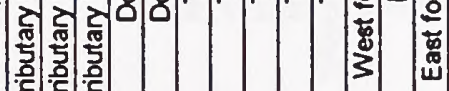

ப்ப்ப்ப்ப்ப்

\& $\$$ : $\$$ 

m.0.0. 0 m.

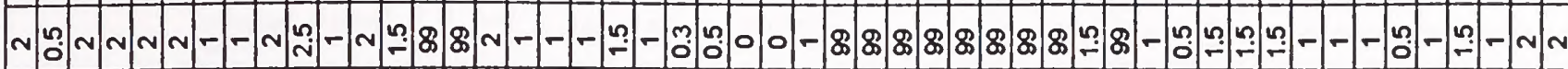

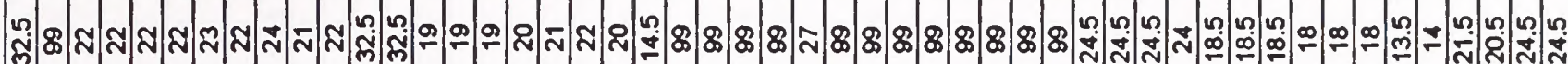

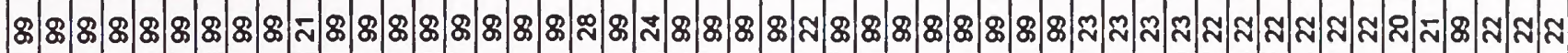
- T: n-f-- - -

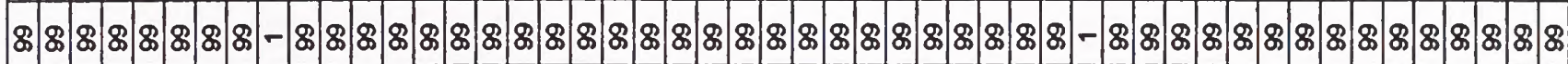

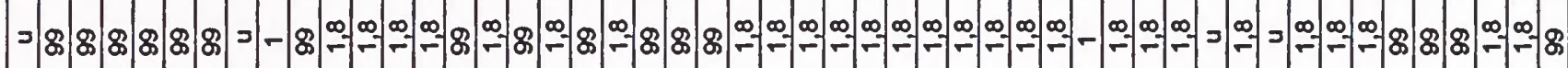

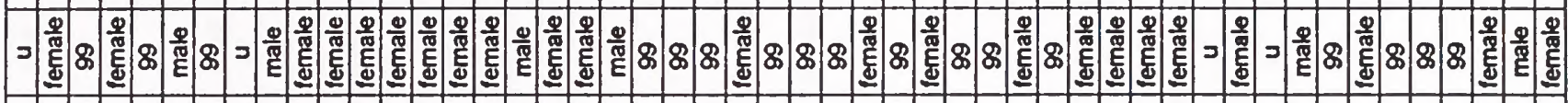

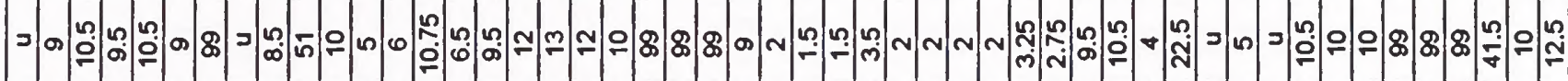

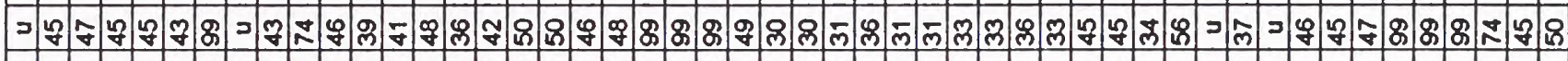

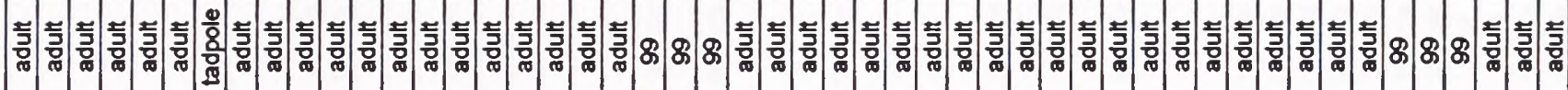
- - - - - - - - - - - - - - - - - - $8 \$ 8-$

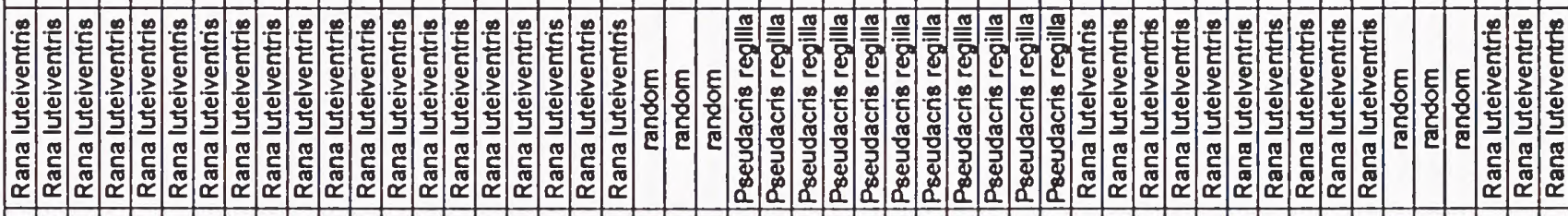

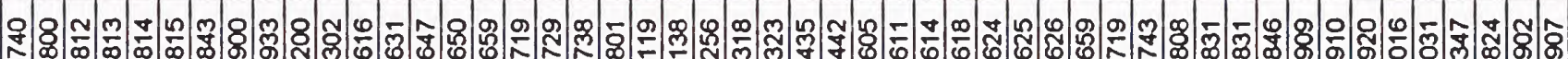

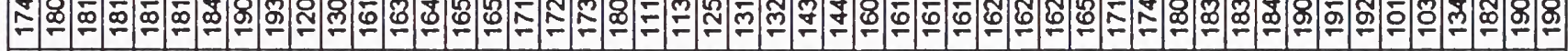

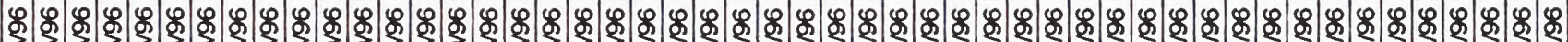

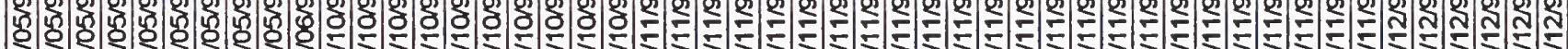

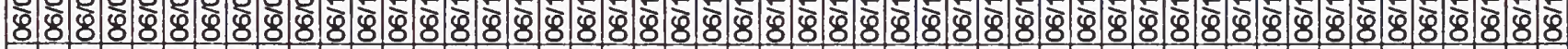

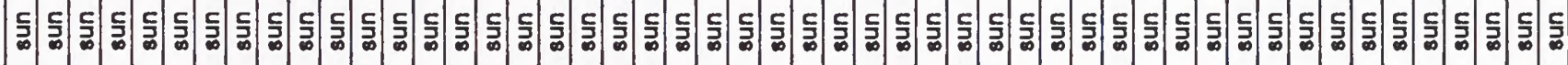

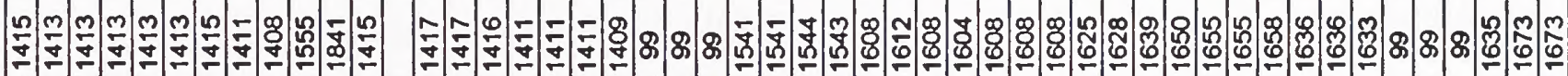
- 7888 等

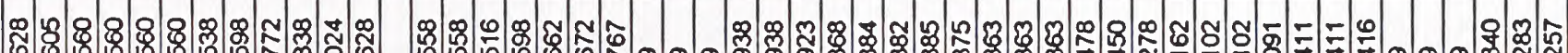

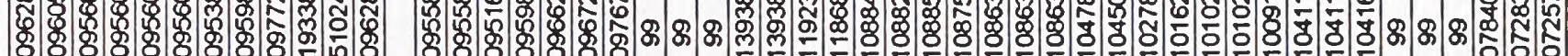

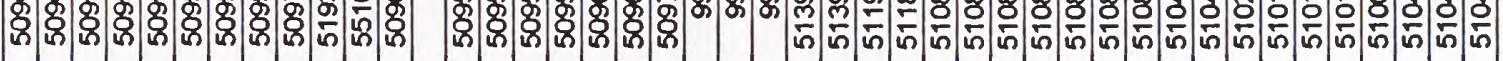

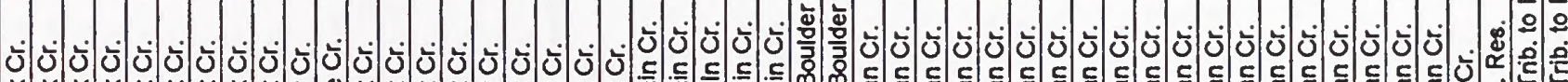
근

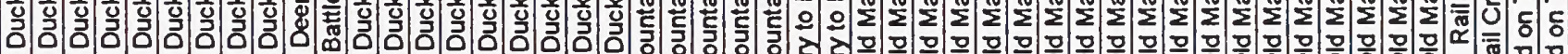

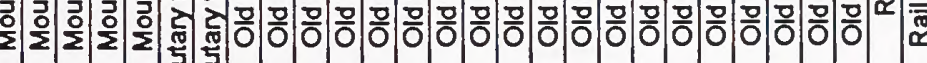



O- O.

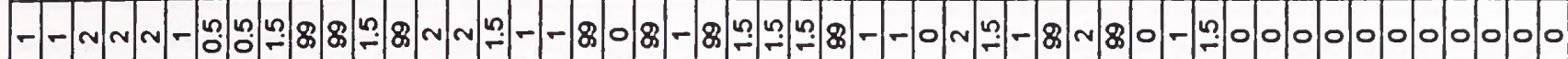
ก.

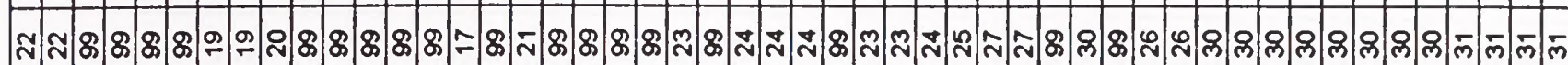
惹

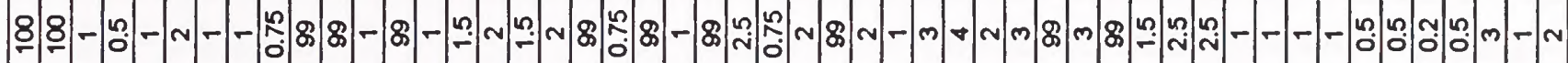
В

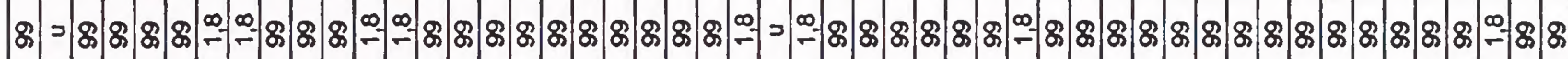

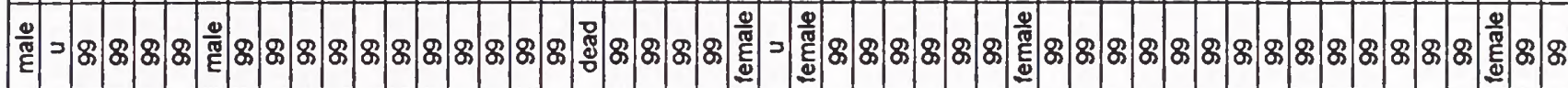

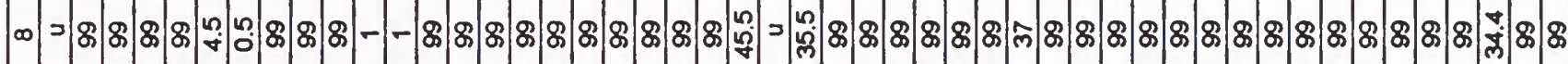

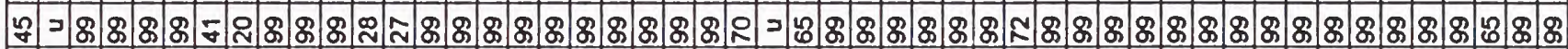
言

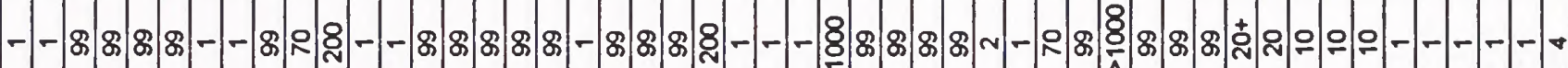

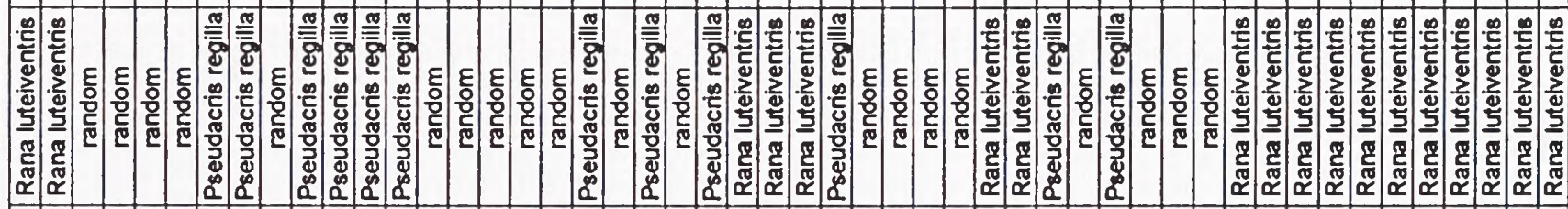

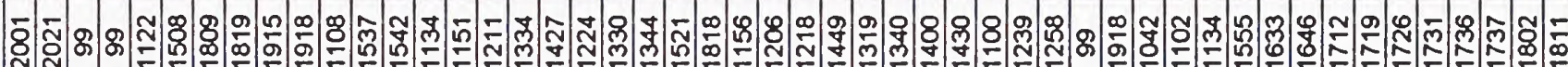
\$ సᄀ

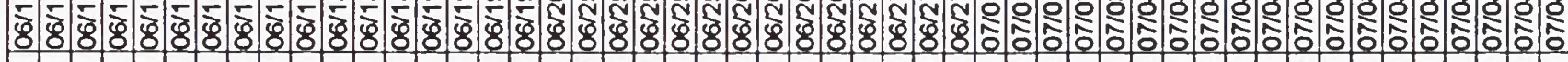

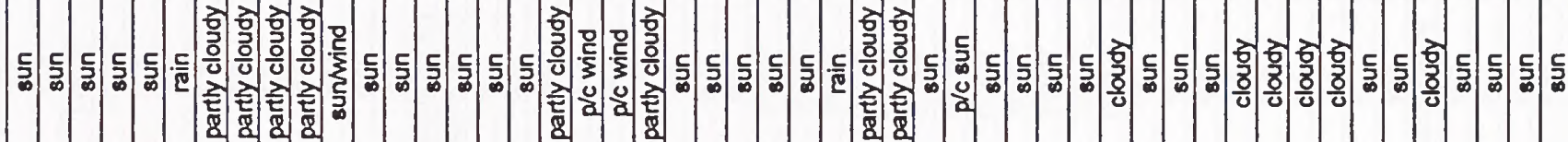

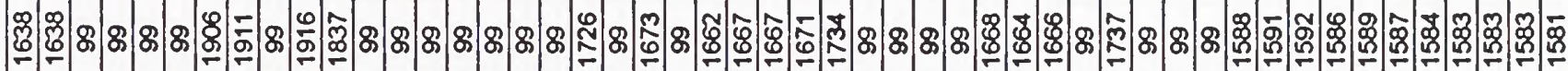

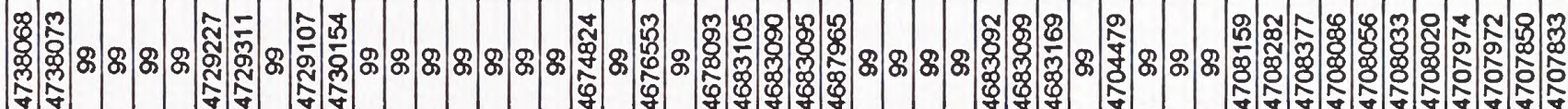
急

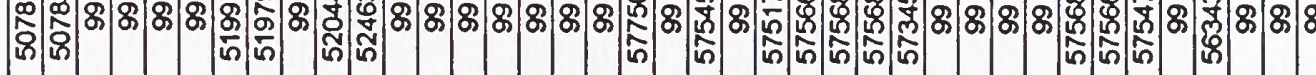

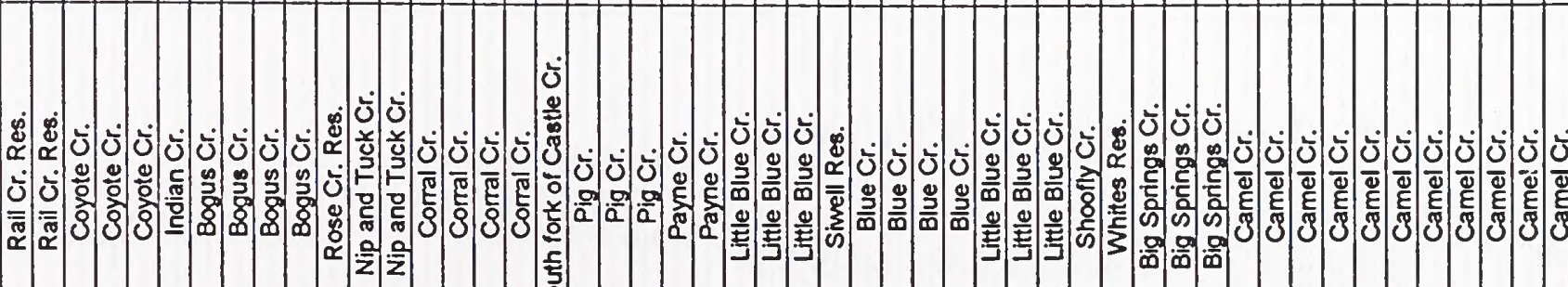





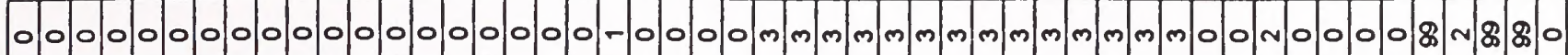
$000000000000-0000000-0-0-0-00-0000-8 \%-$ สงลสก

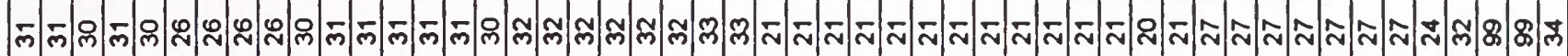

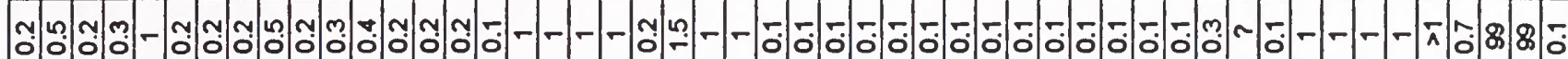

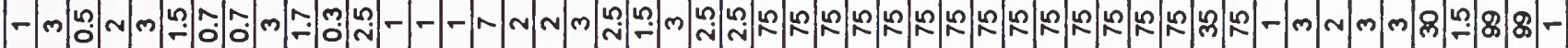

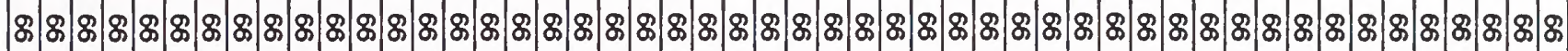

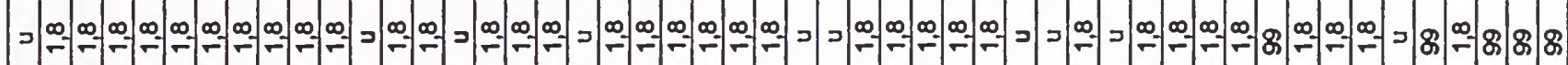

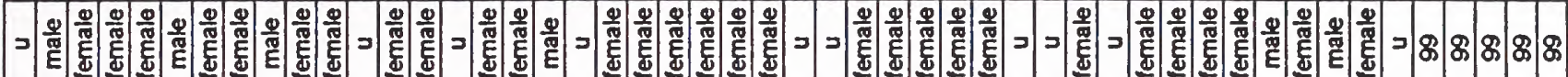

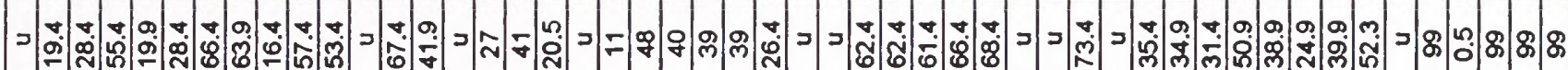
z

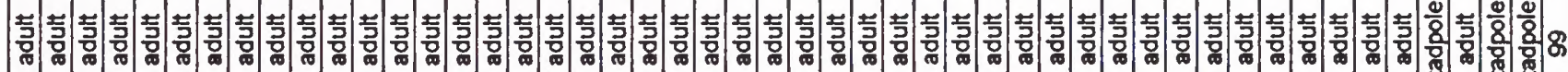

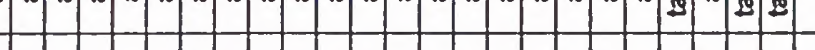

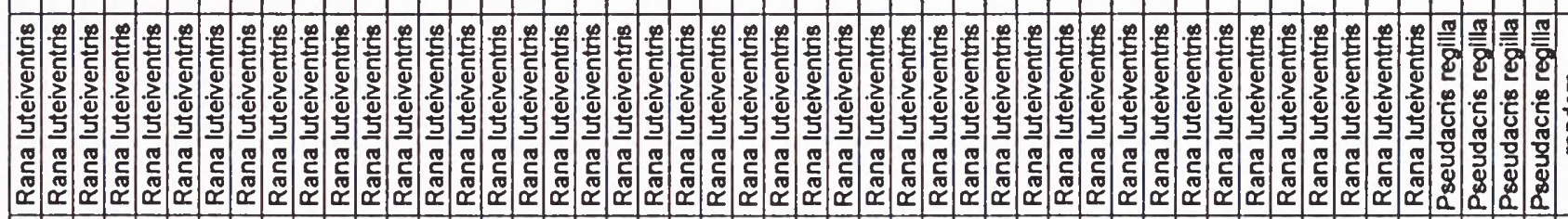

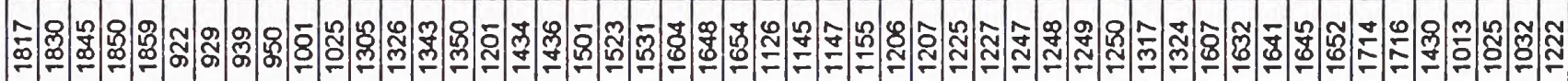

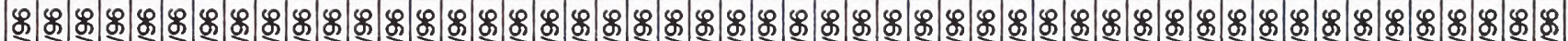
ஜ ஜ

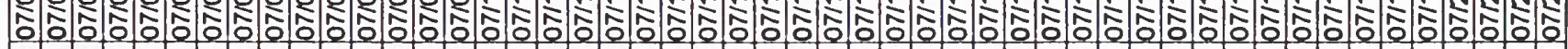

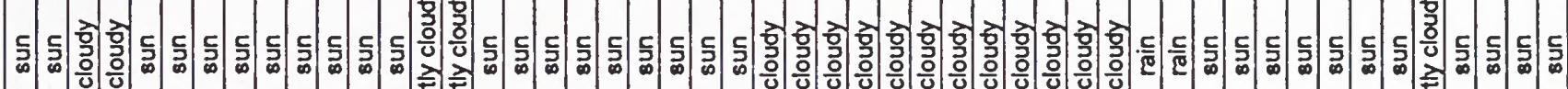
당잉

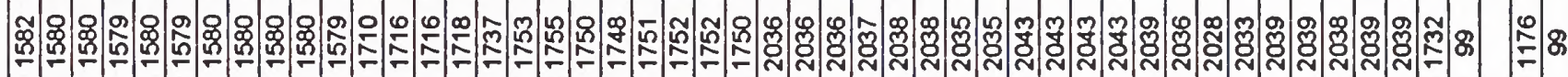

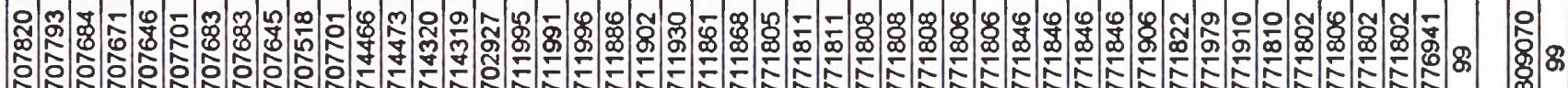

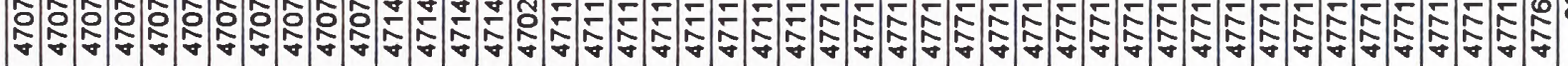

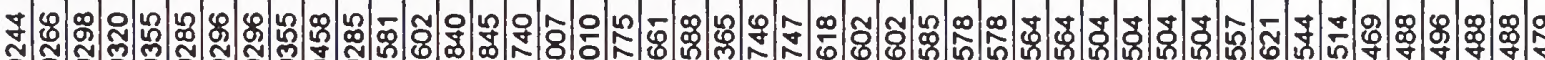

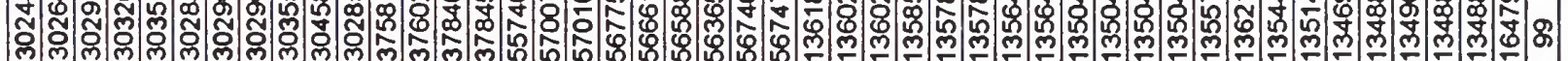

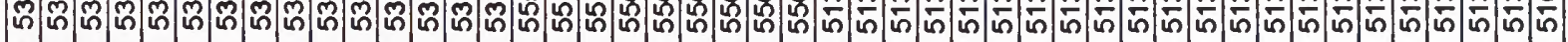

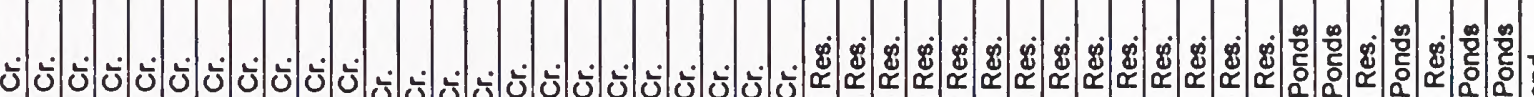

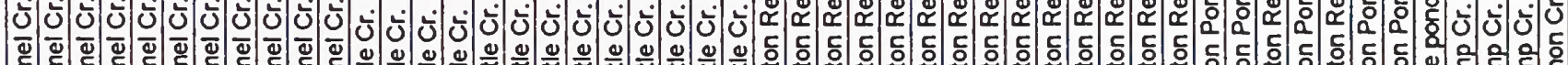

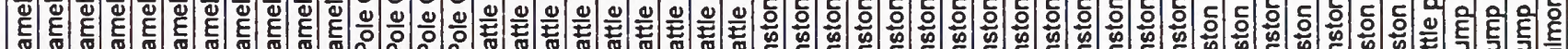
ऽ̄ (1) 



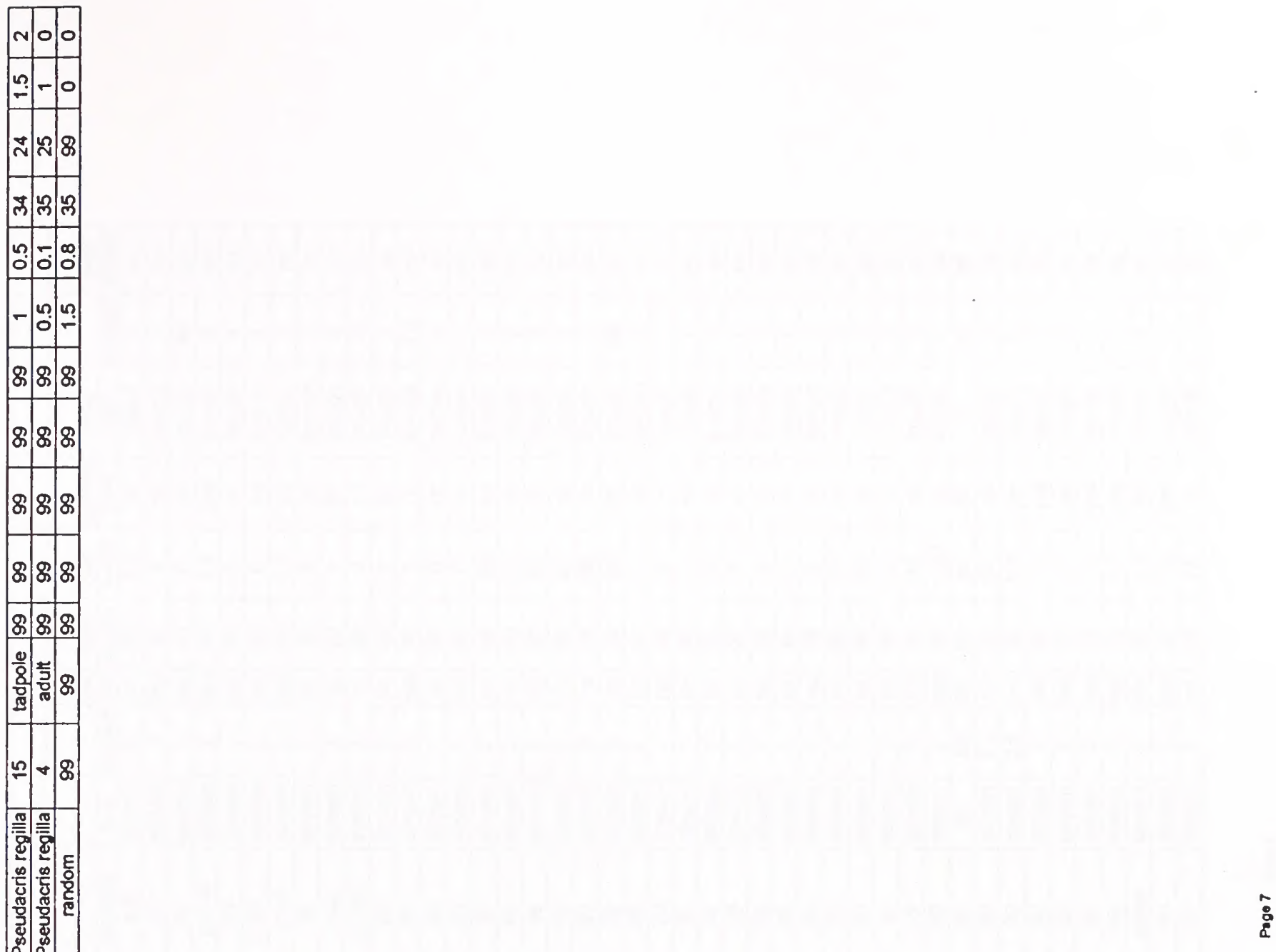
(

$\Rightarrow=$
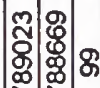
5

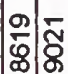

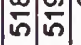





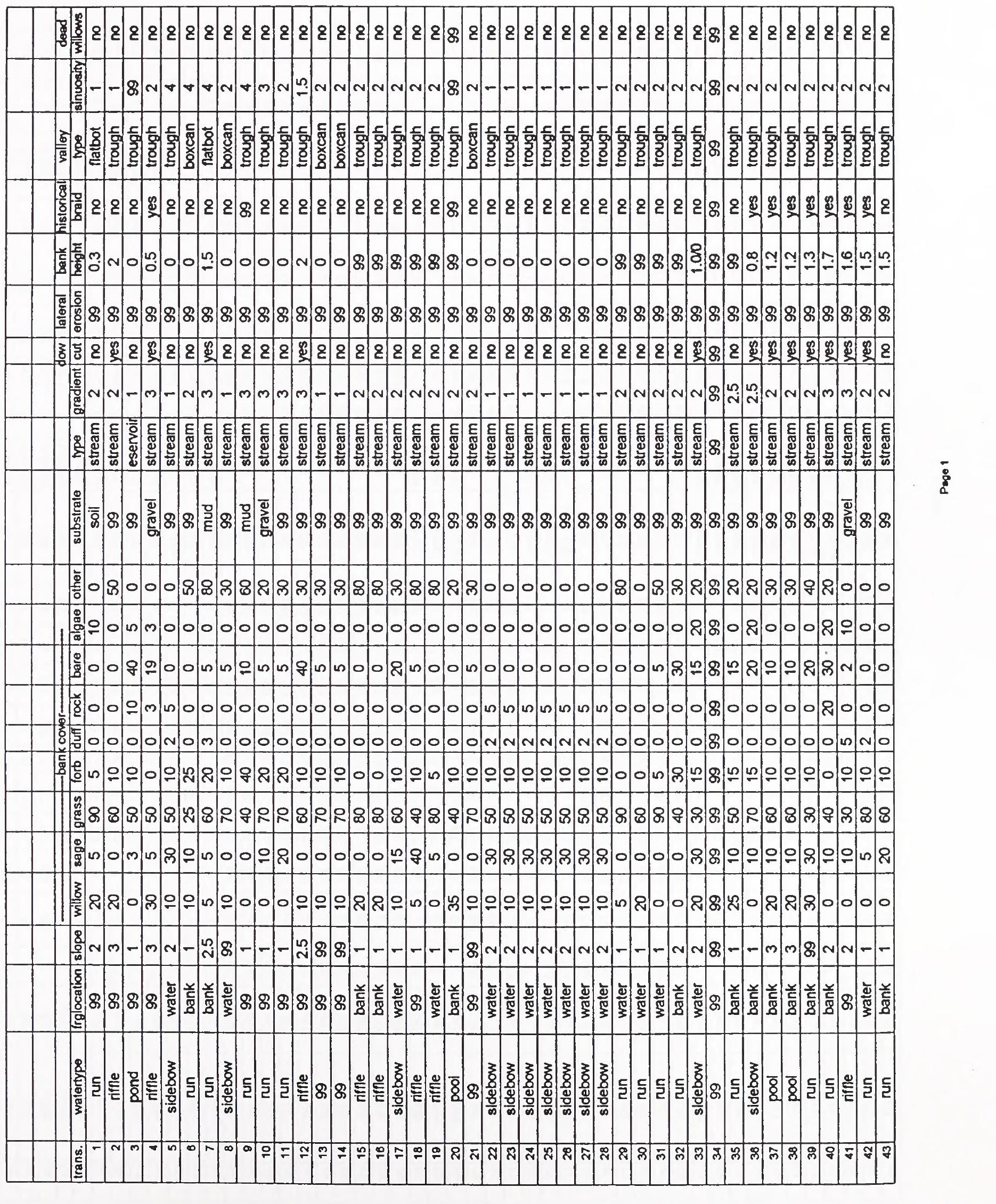





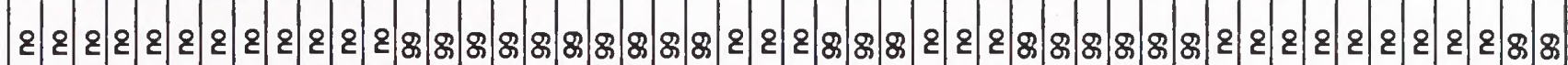

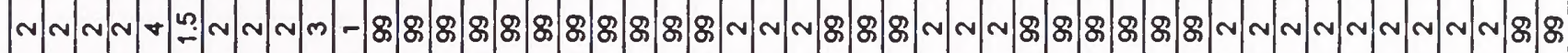

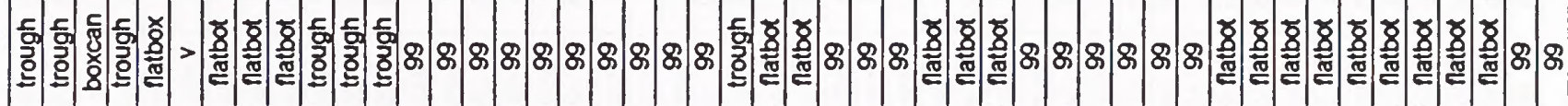

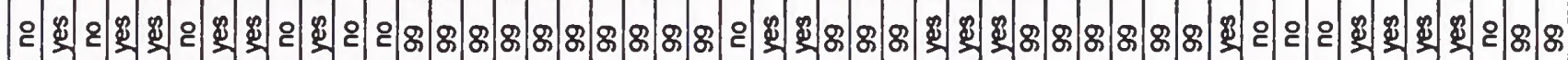

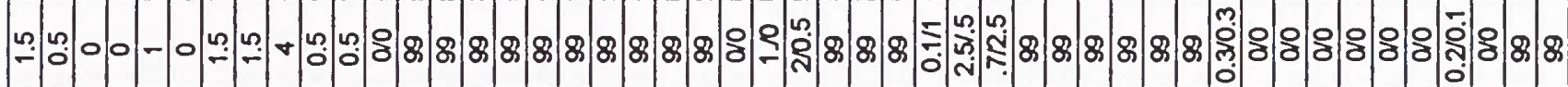
৪ ৪ இ

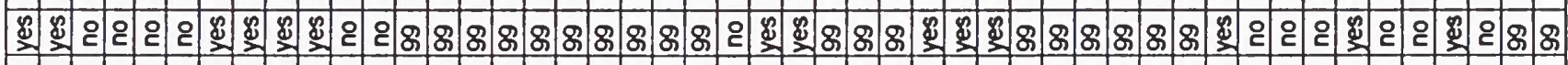

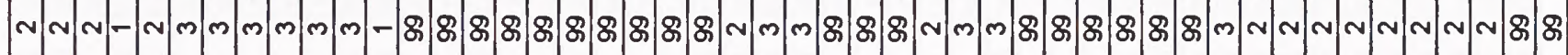

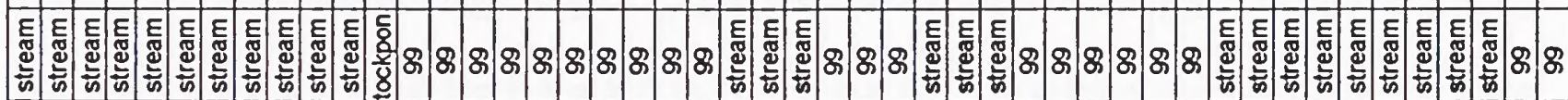

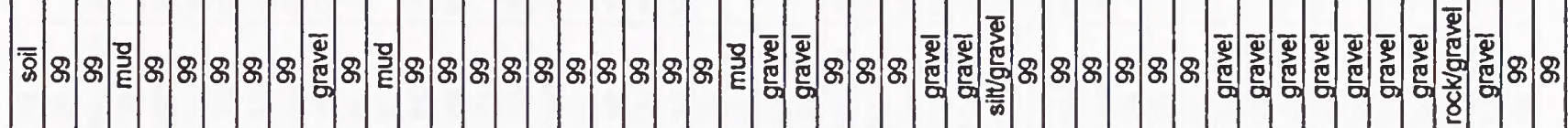

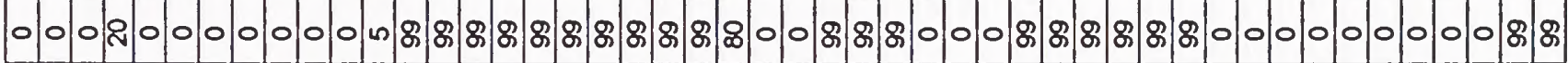
8)

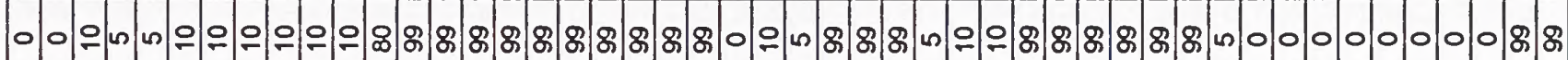

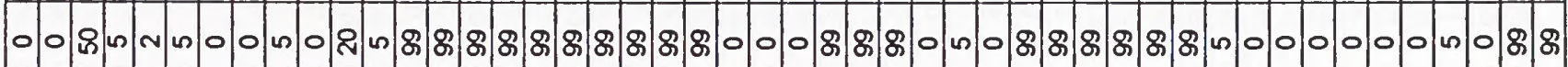

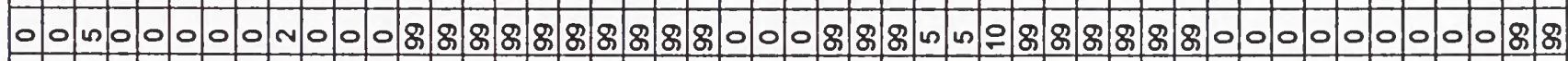

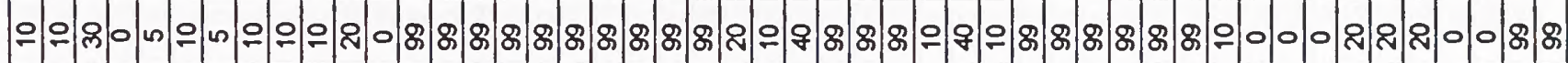

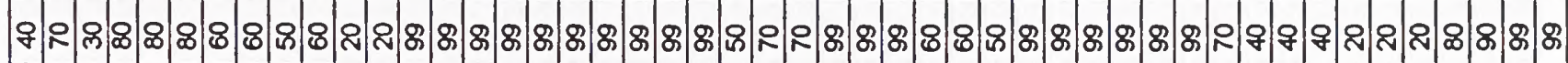

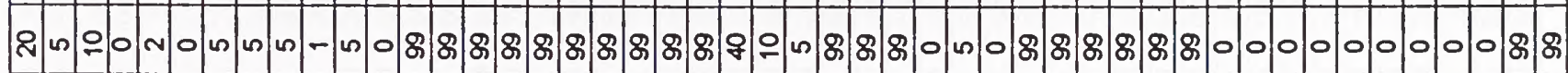
- n 4 ৪

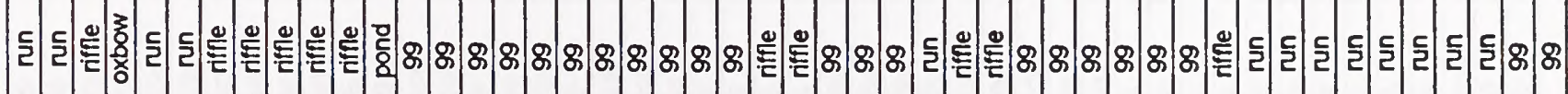





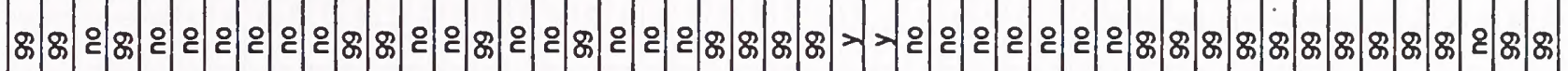
8.8. 8 Nm-

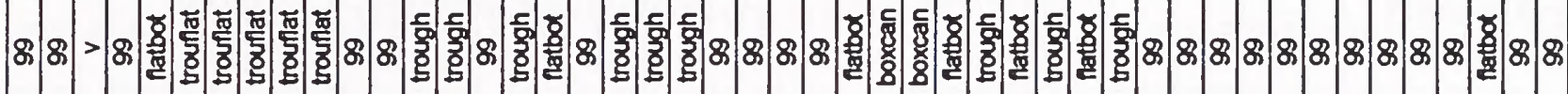

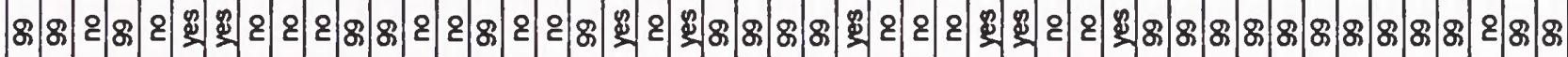

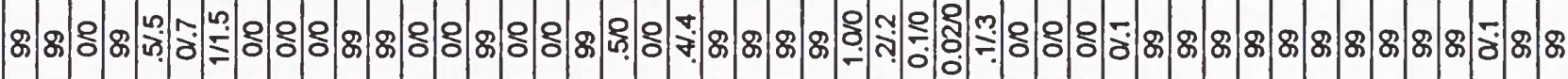

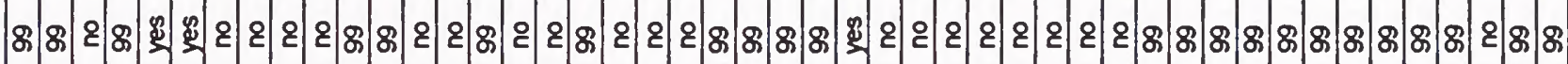

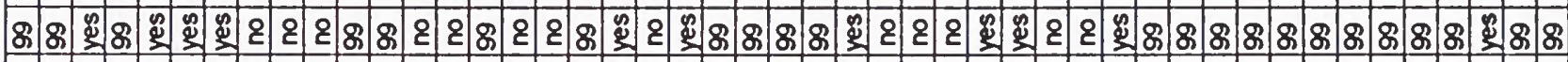
88 m.

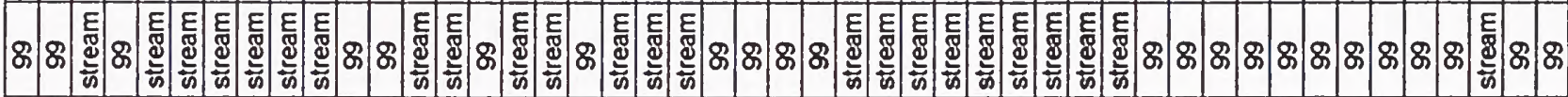

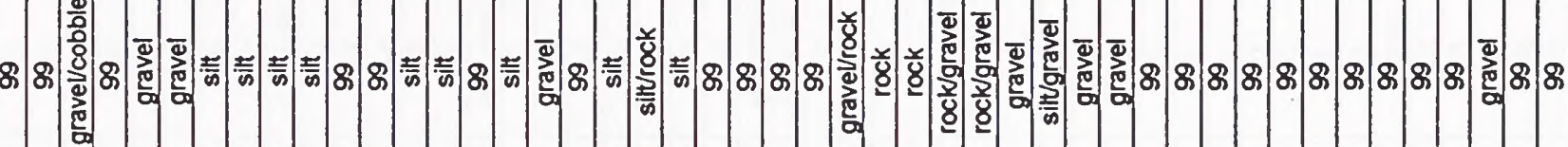

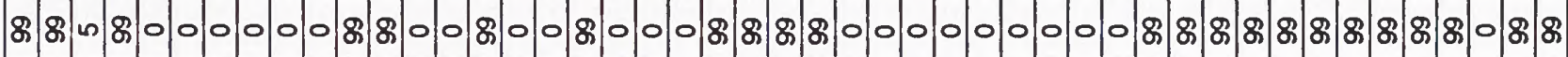

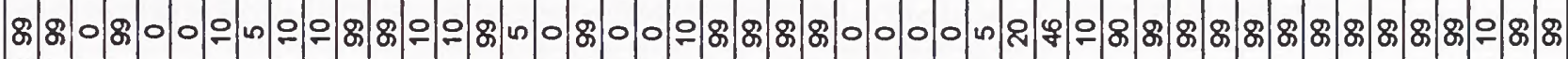

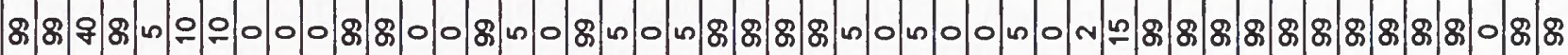

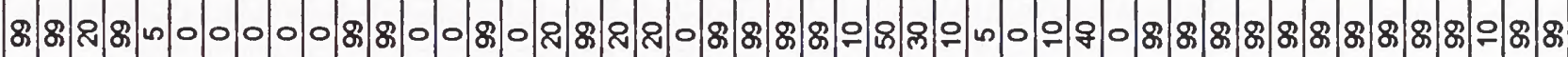

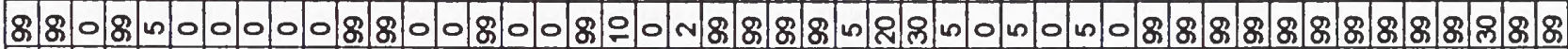

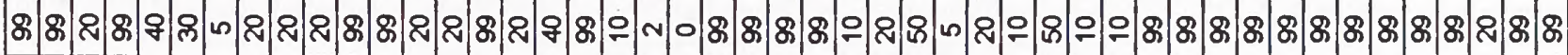

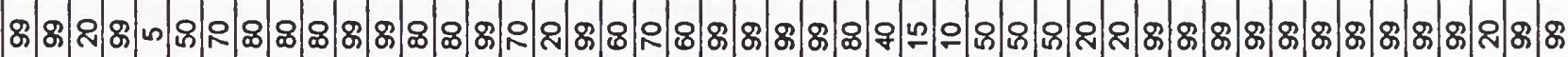

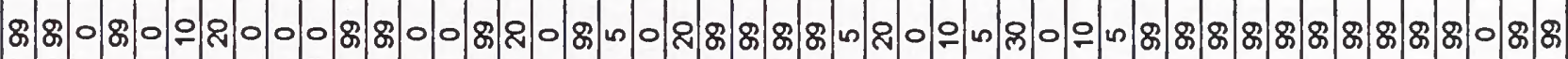

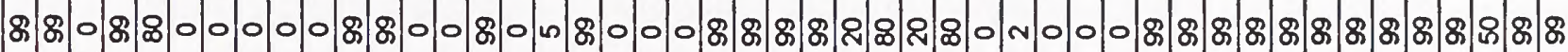

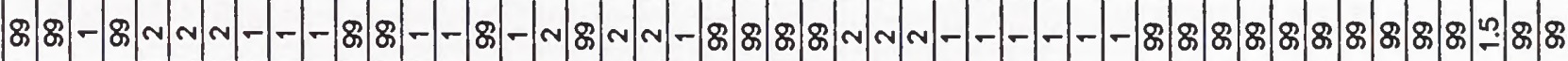

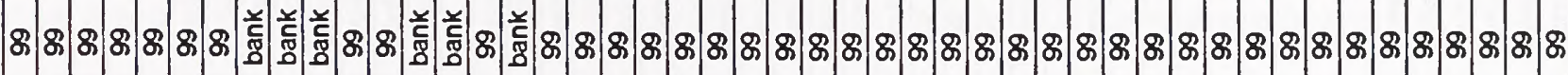
8. 

8

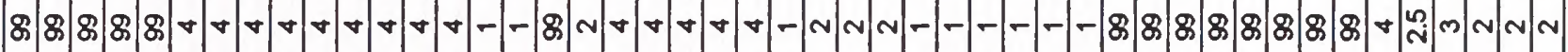

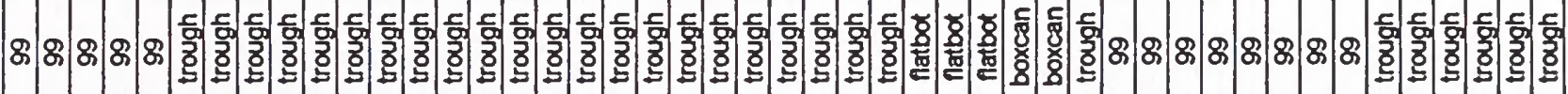
8 8 8 8 \& \&

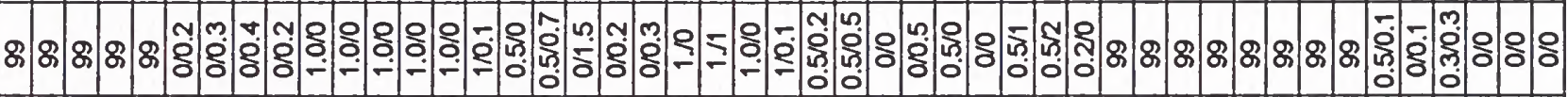

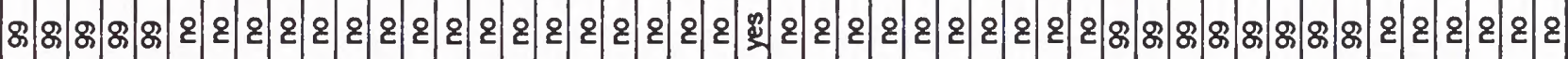

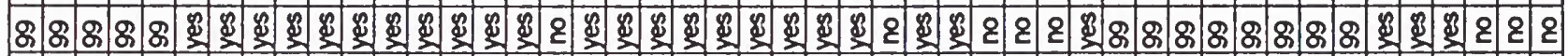

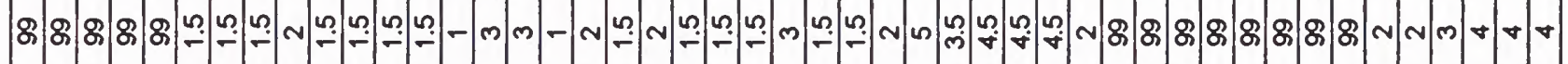

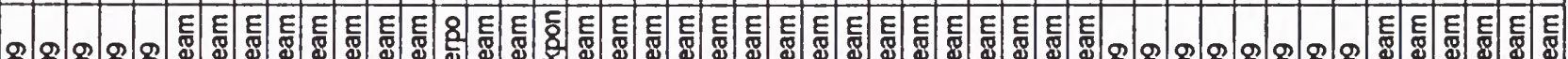

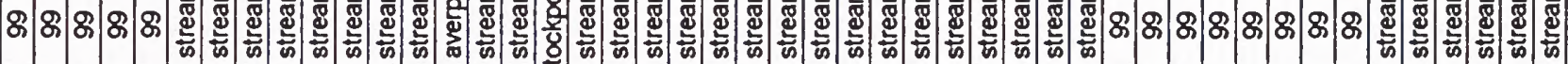

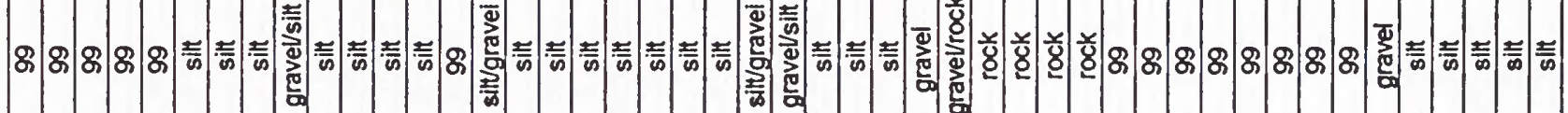

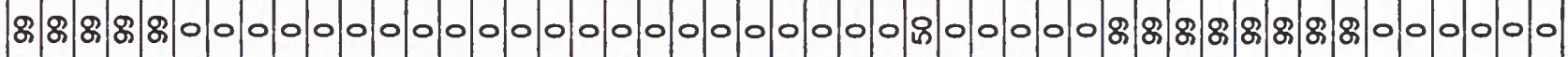

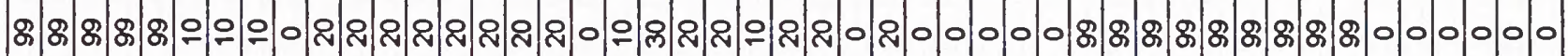

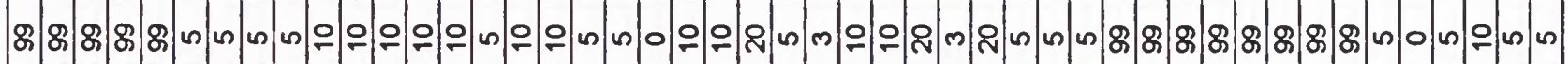

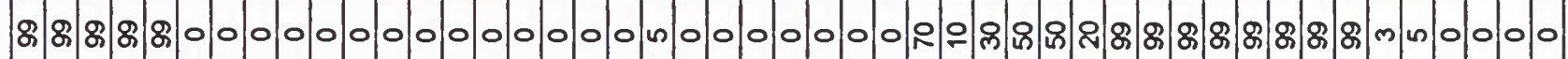

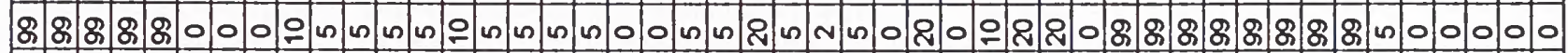

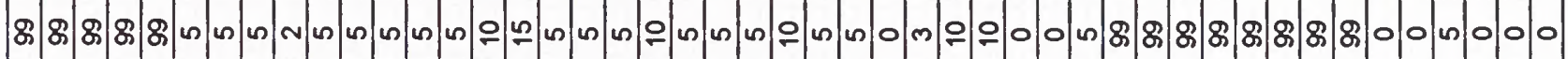

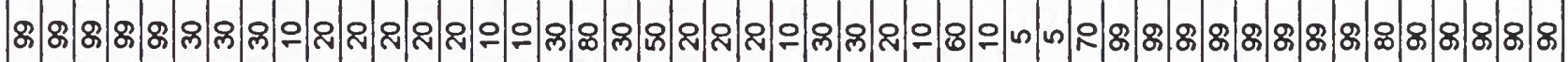

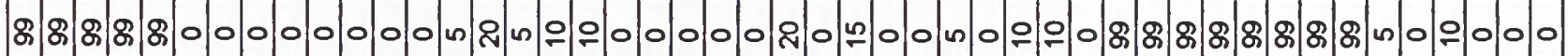

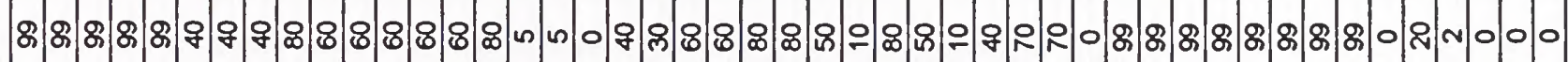

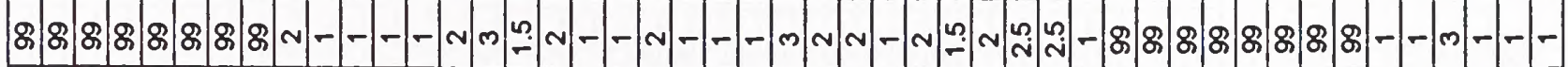

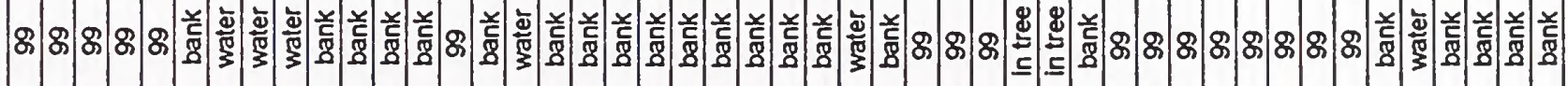

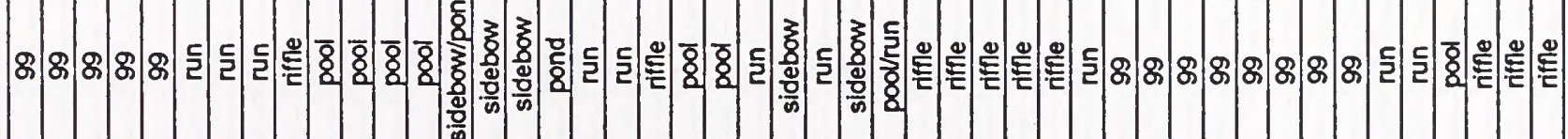





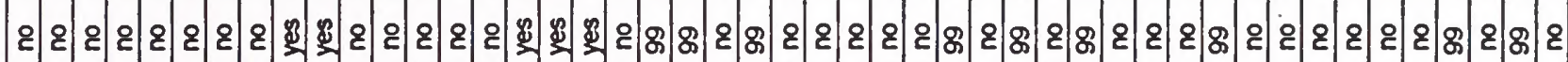

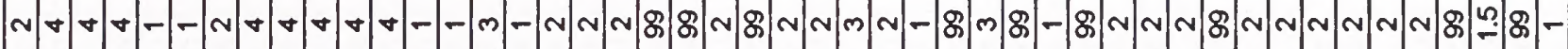

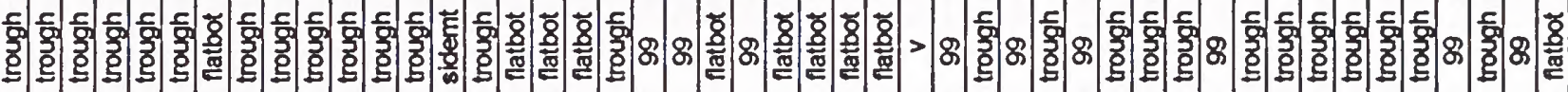

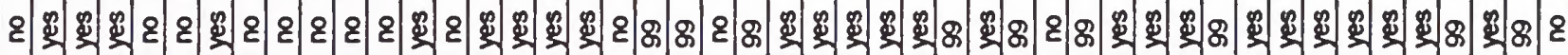

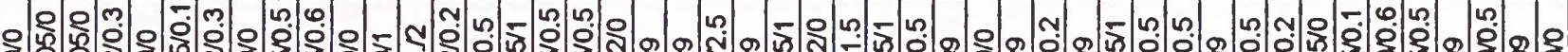
ஓ

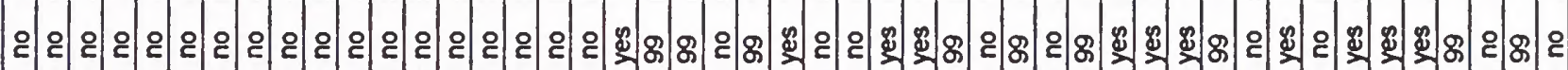

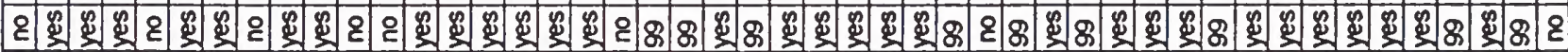
- n n m

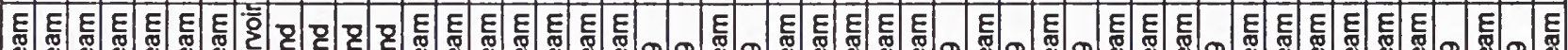

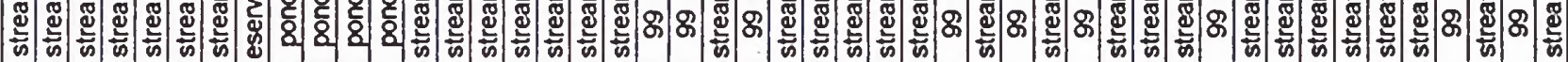

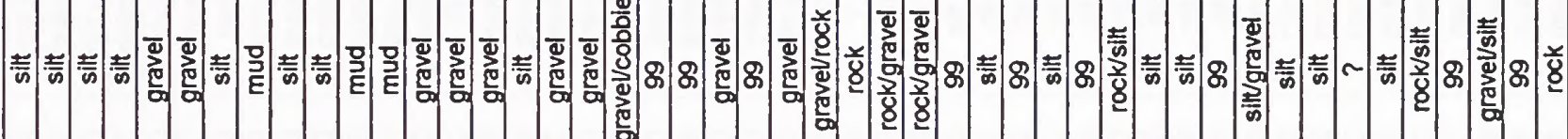

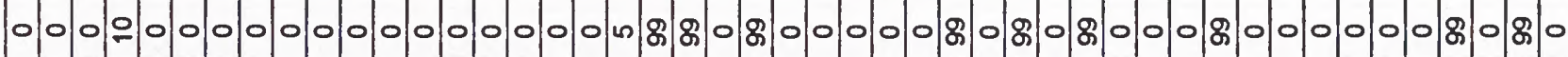
0.000 .0 .8 .

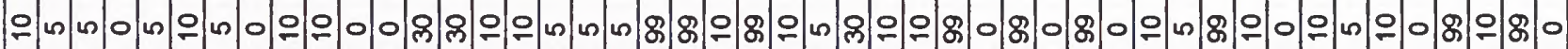

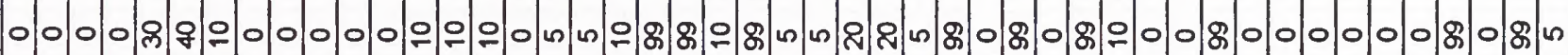

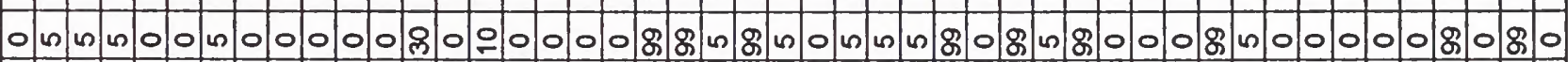

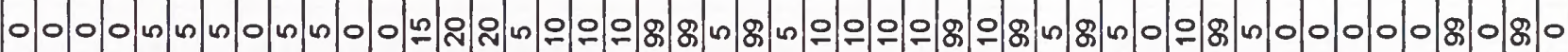

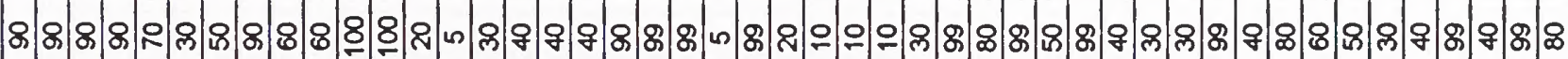

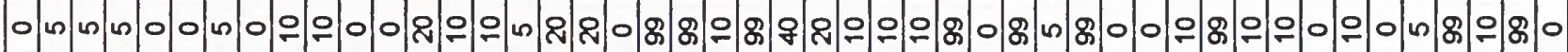

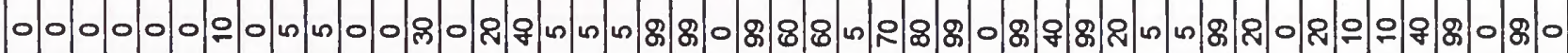

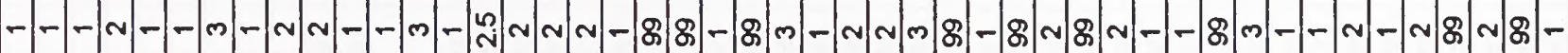

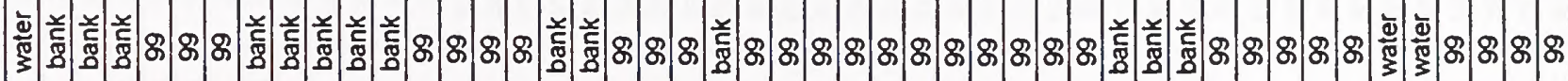
产들 



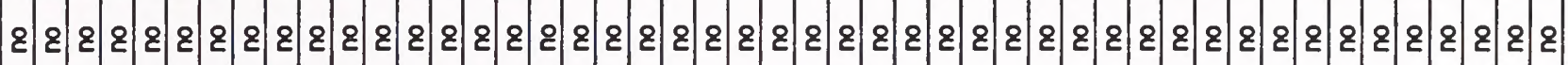

- - -

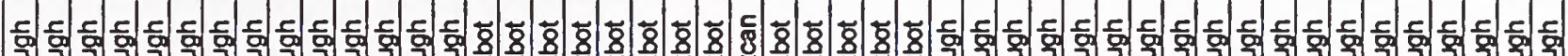

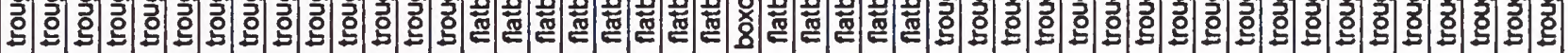

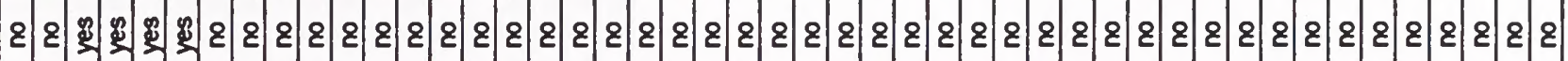

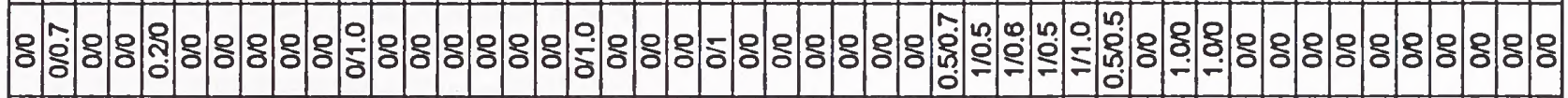

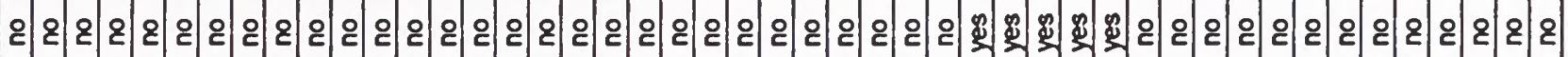

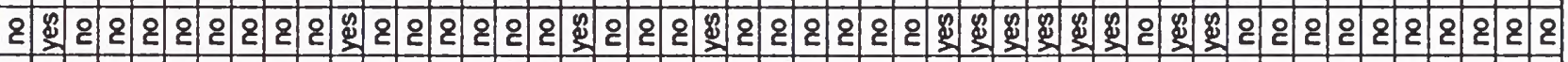

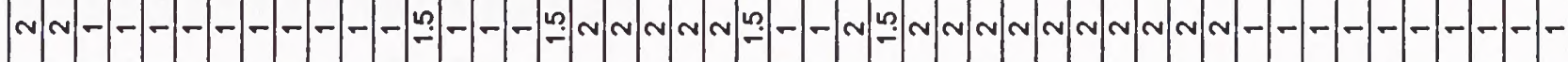

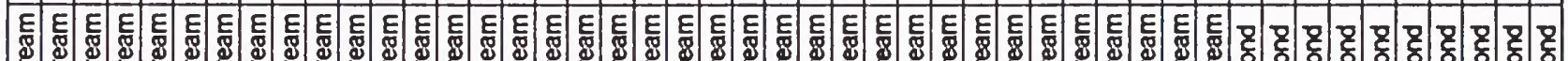

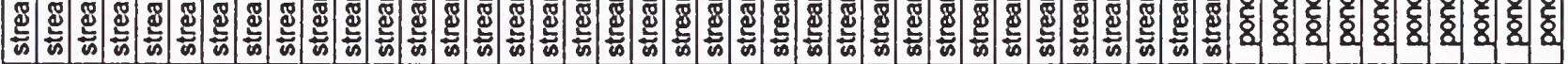

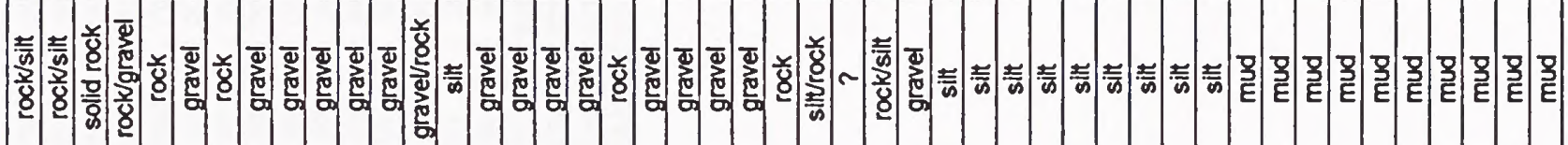

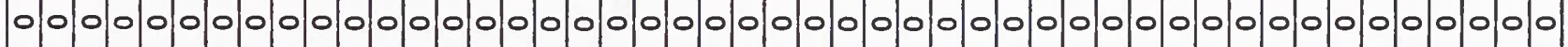

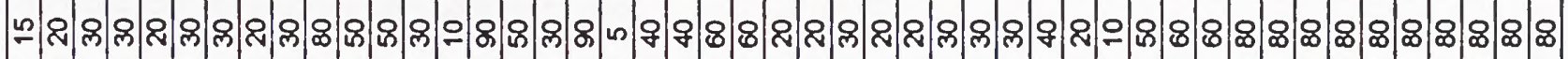

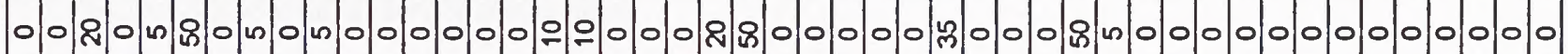

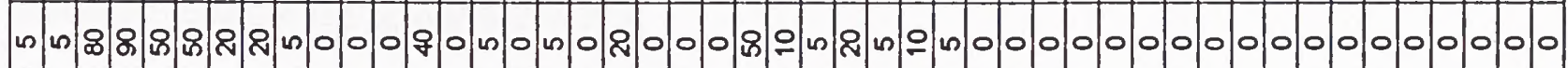

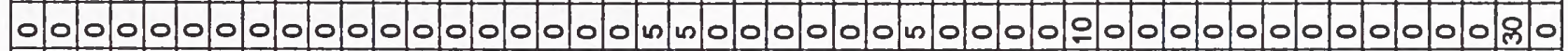

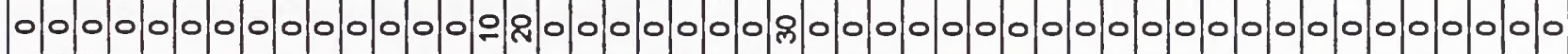
8.

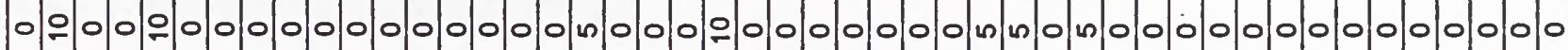

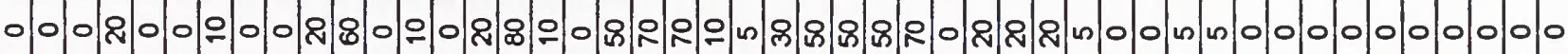

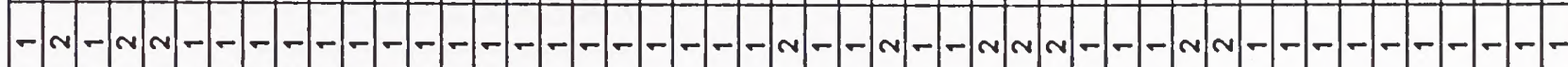

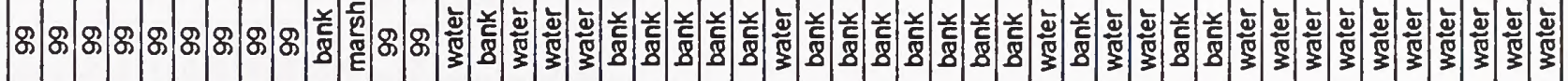

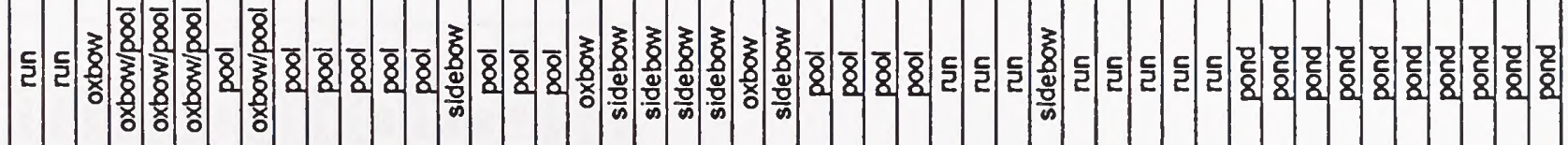

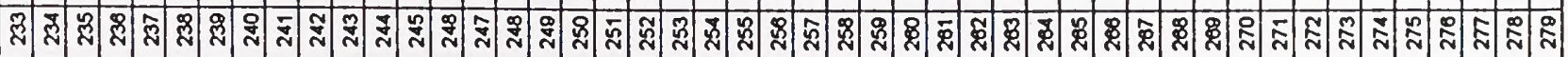





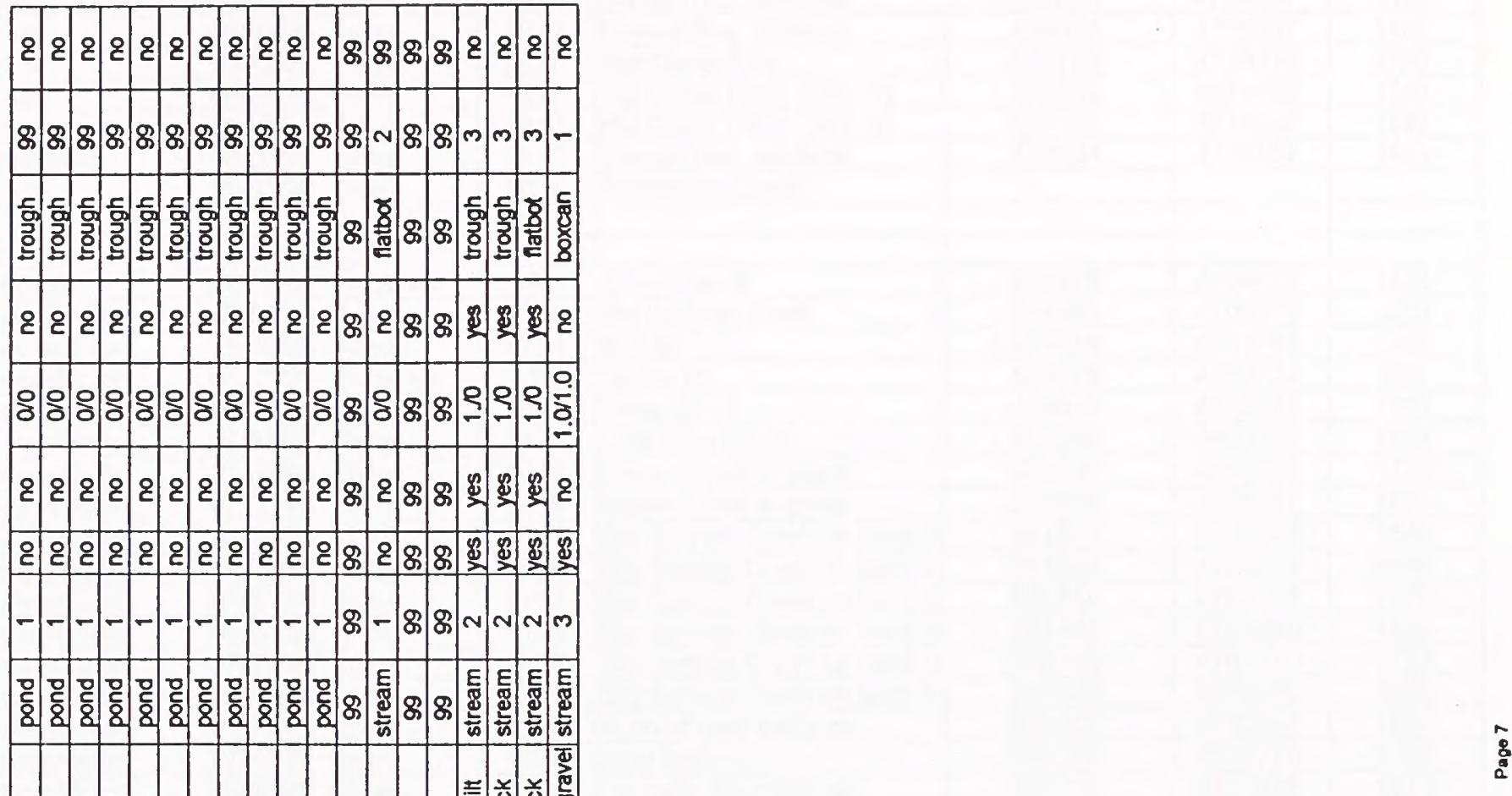

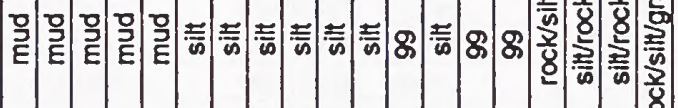

- 0.000000000 .80000

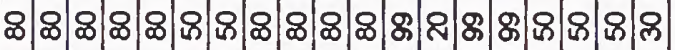

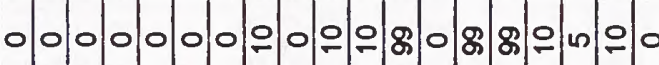

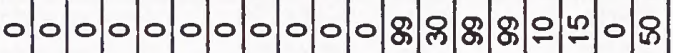

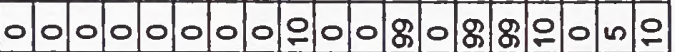

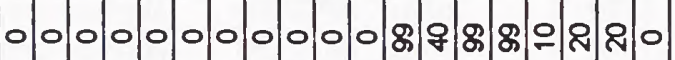

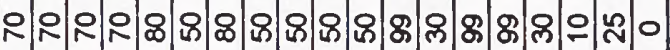
- $000001000 \%$ \&

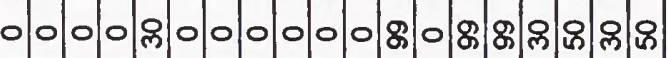

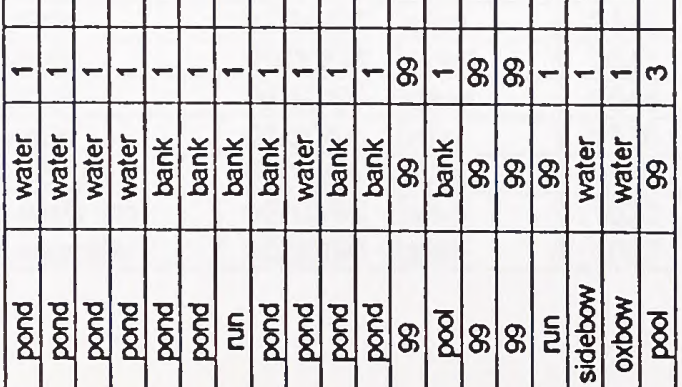

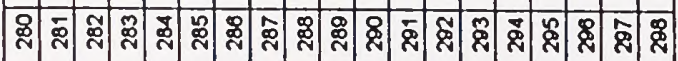





\begin{tabular}{|c|c|c|c|c|c|c|c|}
\hline Appendix II. Incidenta & tures of & phibians & Reo & & & & \\
\hline Genus and Species & date & status & time & locality & UTM & UTM & elevation \\
\hline Amphibians & & & & & NOTE:UTM's and & levation are & incorrected \\
\hline Bufo boreas & $06 / 03 / 96$ & alive & 1816 & Dougal Res. sidebow & 499730 & 4723370 & 1497 \\
\hline Bufo boreas & $06 / 03 / 96$ & alive & 1820 & Dougal Res. sidebow & 499775 & 4723348 & 1459 \\
\hline Bufo boreas & $06 / 03 / 96$ & alive & 1827 & Dougal Res. sidebow & 499699 & 4723440 & 1528 \\
\hline Bufo boreas & $06 / 03 / 96$ & alive & 1837 & Dougal Res. sidebow & 499704 & 4723449 & 1582 \\
\hline Bufo boreas & $06 / 03 / 96$ & alive & 1913 & Dougal Res. sidebow & 499924 & 4723507 & 1463 \\
\hline Bufo boreas & $06 / 03 / 96$ & alive & 1913 & Dougal Res. sidebow & 499924 & 4723507 & 1463 \\
\hline Bufo boreas & $06 / 12 / 96$ & alive & 2027 & Rail Creek Res. & 507785 & 4738316 & 1723 \\
\hline Bufo boreas & $06 / 25 / 96$ & alive & 1212 & Pig Creek Pond, Hwy. 51 & 577530 & 4674988 & 1553 \\
\hline Bufo boreas & $06 / 25 / 96$ & alive & 1212 & Pig Creek Pond, Hwy. 51 & 577530 & 4674988 & 1553 \\
\hline Bufo boreas(tad) & $06 / 03 / 96$ & alive & 1913 & Dougal Res. sidebow & 499924 & 4723507 & 1463 \\
\hline Bufo boreas & $08 / 01 / 96$ & alive & 1037 & Stoneman Creek. & & & \\
\hline & & & & & & & \\
\hline Reptiles & & & & & & & \\
\hline Charina boltae & $07 / 15 / 96$ & road kill & 933 & Silver City rd. & 526278 & 4765445 & 1438 \\
\hline Coluber constrictor & $07 / 04 / 96$ & alive & 1034 & Big Springs Creek & 551565 & 4702875 & 1562 \\
\hline Coluber constrictor & $05 / 30 / 96$ & alive & 1734 & M.F.R. & 555574 & 4731780 & 1505 \\
\hline Coluber constrictor & $06 / 03 / 96$ & road kill & 809 & Simcord & 584778 & 4780334 & 892 \\
\hline Coluber constrictor & $06 / 04 / 96$ & road kill & 1815 & Trout ck. rd. & 499699 & 4756574 & 1362 \\
\hline Coluber constrictor & $07 / 01 / 96$ & alive & 1308 & Little Blue Creek & 573286 & 4683381 & 1625 \\
\hline Coluber constrictor & $07 / 02 / 96$ & alive & 1817 & Indian Creek in grass & 575447 & 4668218 & 1724 \\
\hline Coluber constrictor & $07 / 02 / 96$ & alive & 1836 & Indian Creek in grass & 575006 & 4668397 & 1900 \\
\hline Coluber constrictor & $07 / 04 / 96$ & alive & 1053 & Big Springs Creek on bank in & 551552 & 4702236 & 1640 \\
\hline Coluber constrictor & $07 / 04 / 96$ & alive & 1057 & Big Springs Creek on bank in & 551582 & 4702256 & 1696 \\
\hline Coluber constrictor & $07 / 04 / 96$ & alive & 1100 & Big Springs Croek on bank in & 551580 & 4702190 & 1778 \\
\hline Coluber constrictor & $07 / 04 / 96$ & alive & 1118 & Big Springs Creek on bank in & 551498 & 4702028 & 1645 \\
\hline Coluber constrictor & $07 / 04 / 96$ & alive & 1200 & Big Springs Creek on bank in & 552100 & 4701170 & 1720 \\
\hline Coluber constrictor & $07 / 04 / 96$ & alive & 1204 & Big Springs Creek on bank in & 552396 & 4700589 & 1856 \\
\hline Coluber constrictor & $07 / 10 / 96$ & alive & 1107 & in pond near battle ck. & 556203 & 4702054 & 1659 \\
\hline Coluber constrictor & $07 / 10 / 96$ & alive & 1148 & near battle ck. & 555863 & 4702770 & 1787 \\
\hline Coluber constrictor & $07 / 10 / 96$ & alive & 1222 & on bank, Reynolds ck. & 519776 & 4777283 & 1275 \\
\hline Coluber constrictor & $06 / 11 / 96$ & alive & 1334 & Mountain Creek & 513884 & 4742021 & 1511 \\
\hline Coluber constrictor & $06 / 11 / 96$ & alive & 1340 & Mountain Creek & 513798 & 4741946 & 1516 \\
\hline Coluber constrictor & $06 / 12 / 96$ & alive & 1050 & Old Man Creek & 511151 & 4741920 & 1353 \\
\hline Coluber constrictor & $06 / 19 / 96$ & Road kill & 1835 & Indian Meadows road & 506323 & 4741051 & 1603 \\
\hline Coluber constrictor & $06 / 25 / 96$ & Road kill & 849 & Mud Flat road & 555976 & 4732118 & 1472 \\
\hline Coluber constrictor & $06 / 25 / 96$ & alive & 1237 & Pig Creek Pond, Hwy. 51 & 577546 & 4675000 & 1713 \\
\hline Coluber constrictor & $07 / 24 / 96$ & alive & 945 & on bank of Salmon Cr. & 536197 & 4791523 & 1440 \\
\hline Coluber constrictor & $07 / 25 / 96$ & alive & 1438 & Little Hardtrigger $\mathrm{Cr}$. on bank. & 516484 & 4799449 & 995 \\
\hline Coluber constrictor & $07 / 30 / 96$ & road kill & 953 & Mud Flat road & 551585 & 4728665 & 1969 \\
\hline Cnemidophorus tignis & $07 / 11 / 96$ & alive & 1042 & M.F.R. & 574237 & 4744829 & 853 \\
\hline Cnemidophorus tignis & $07 / 11 / 96$ & alive & 1310 & M.F.R. & 547441 & 4767689 & 1268 \\
\hline Cnemidophorus tignis & $07 / 11 / 96$ & road kill & 1754 & Jordan Crater rd. & 476525 & 4777972 & 1348 \\
\hline Cnemidophorus tignis & $06 / 10 / 96$ & alive & 1011 & Fossil Butte & 545632 & 4773111 & 876 \\
\hline Cnemidophorus tigris & $06 / 10 / 96$ & alive & 1021 & Fossil Butte & 545023 & 4772749 & 936 \\
\hline Cnemidophorus tignis & $07 / 25 / 96$ & alive & 1440 & Road to Hardtrigger. & 519654 & 4803173 & 979 \\
\hline Crotalus viridis & $06 / 04 / 96$ & road kill & 1810 & Trout ck. rd. & 499950 & 4756822 & 1390 \\
\hline Crotalus viridis & $06 / 04 / 96$ & alive & 1845 & Indian Meadows rd. & 506984 & 4748913 & 1635 \\
\hline Crotalus vinidis & $06 / 04 / 96$ & alive & 2040 & Jordan Valley ck. & 509952 & 4753399 & 1537 \\
\hline Crotalus vinidis & $07 / 15 / 96$ & road kill & 856 & Silver City rd. & 533032 & 4772327 & 1149 \\
\hline Crotalus viridis & $07 / 15 / 96$ & alive & 917 & Silver City rd. & 530908 & 4768395 & 1123 \\
\hline Crotalus viridis & $07 / 16 / 96$ & alive & 1143 & on rocks, Reynolds ck. & 520013 & 4778255 & 1178 \\
\hline Crotalus viridis & $06 / 06 / 96$ & dead & 2047 & Simco road & 584610 & 4781571 & 905 \\
\hline Crotalus viridis & $06 / 10 / 96$ & dead & 1548 & Flint Creek & 514664 & 4748932 & 1511 \\
\hline Crotalus viridis & $07 / 30 / 96$ & alive & 1736 & Castle $\mathrm{Cr}$. under sagebrush. & 527564 & 4695524 & 1685 \\
\hline Gambelia wislizenii & $06 / 10 / 95$ & alive & 954 & Fossil Butte & 545090 & 4772730 & 953 \\
\hline Gambelia wislizenii & $06 / 10 / 96$ & alive & 1022 & Fossil Butte & 545023 & 4772749 & 936 \\
\hline Pituophis catenifer & $05 / 30 / 96$ & alive & 1802 & M.F.R. & 575251 & 4752168 & 566 \\
\hline
\end{tabular}





\begin{tabular}{|c|c|c|c|c|c|c|c|}
\hline Pituophis catenifer & $05 / 30 / 96$ & alive & 1828 & M.F.R. & 575349 & 4753566 & 980 \\
\hline Pituophis catenifer & $05 / 30 / 96$ & alive & 1831 & M.F.R. & 575414 & 4750209 & 811 \\
\hline Pituophis catenifer & $05 / 30 / 96$ & alive & 1832 & M.F.R. & 575355 & 4750144 & 826 \\
\hline Pituophis catenifer & $06 / 03 / 96$ & alive & 821 & Simcord & 584644 & 4780334 & 880 \\
\hline Pituophis catenifer & $06 / 03 / 96$ & alive & 834 & Simco rd & 584072 & 4771753 & 885 \\
\hline Pituophis catenifer & $06 / 03 / 96$ & road kill & 905 & M.F.R. & 575.370 & 4750427 & 745 \\
\hline Pituophis catenifer & $06 / 03 / 96$ & road kill & 910 & M.F.R. & 574777 & 4747531 & 677 \\
\hline Pituophis catenifer & $06 / 04 / 96$ & alive & 1049 & Trout ck. & 503410 & 4756753 & 1390 \\
\hline Pituophis catenifer & $06 / 04 / 96$ & alive & 1751 & Trout ck. rd. & 502997 & 4756354 & 1366 \\
\hline Pituophis catenifer & $06 / 04 / 96$ & road kill & 1824 & Indian Meadows rd. & 501723 & 4752344 & 1450 \\
\hline Pituophis catenifer & $06 / 04 / 96$ & alive & 1830 & Indian Meadows rd. & 502246 & 4752244 & 1742 \\
\hline Pituophis catenifer & $06 / 04 / 96$ & alive & 1806 & Trout ck. rd. & 500742 & 4757140 & 1012 \\
\hline Pituo phis catenifer & $07 / 08 / 96$ & road kill & 804 & Mud Flat Road & 556382 & 4732736 & 1406 \\
\hline Pituophis catenifer & $07 / 18 / 96$ & road kill & 1040 & De lamar rd. & 502180 & 4756982 & 1422 \\
\hline Pituophis catenifer & $07 / 18 / 96$ & alive & 1215 & Succor ck. on rocks & 502122 & 4780753 & 1242 \\
\hline Pituophis catenifer & $06 / 06 / 96$ & alive & 1738 & Josephine Ranch road & 528994 & 4732726 & 1715 \\
\hline Pituophis catenifer & $06 / 06 / 96$ & alive & 1946 & Mud Flat road & 563113 & 4738726 & 1126 \\
\hline Pituophis catenifer & $06 / 06 / 96$ & alive & 2033 & Simco road & 584050 & 4772944 & 886 \\
\hline Pituophis catenifer & $06 / 06 / 96$ & alive & 2037 & Simco road & 584448 & 4775231 & 792 \\
\hline Pituophis catenifer & $06 / 10 / 96$ & dead & 1837 & Triangle Road & 508864 & 4748040 & 1409 \\
\hline Pituophis catenifer & $06 / 10 / 96$ & dead & 1845 & Triangle Road & 508510 & 4747777 & 1517 \\
\hline Pituophis catenifer & $06 / 17 / 96$ & Road kill & 1809 & Corral Creek road & 513303 & 4724367 & 1801 \\
\hline Pituophis catenifer & $06 / 20 / 96$ & alive & 1425 & South fork of Castle Creek & & & \\
\hline Pituophis catenifer & $07 / 01 / 96$ & Road kill & 827 & Hwy 51 & 601277 & 4756170 & 741 \\
\hline Pituophis catenifer & $07 / 01 / 96$ & Road kill & 827 & Hwy 51 & 601277 & 4756170 & 741 \\
\hline Pituophis catenifer & $07 / 01 / 96$ & Road kill & 835 & Hwy 51, $1 / 2$ mile North of $\mathrm{Bru}$ & & & \\
\hline Pituophis catenifer & $07 / 01 / 96$ & Road kill & 858 & Hwy 51 & 589938 & 4722572 & 1422 \\
\hline Pituophis catenifer & $07 / 30 / 96$ & road kill & 1020 & Mud Flat road & 563810 & 4739103 & 1135 \\
\hline Phrynosoma douglassii & $07 / 01 / 96$ & alive & 1020 & Bybee Res. $10 \mathrm{~m}$ from water & 560851 & 4677928 & 1495 \\
\hline Phrynosoma douglassii & $07 / 11 / 96$ & road kill & 1051 & M.F.R. & 575331 & 4749656 & 768 \\
\hline Phrynosoma douglassii & $06 / 26 / 96$ & alive & 1752 & Patch of short sage and bare & 565557 & 4691812 & 1651 \\
\hline Phrynosoma platyrhinos & $07 / 25 / 96$ & alive & 1005 & Hardtrigger $\mathrm{Cr}$. on bank & 519971 & 4802711 & 953 \\
\hline Sceloporus graciosus & $05 / 28 / 96$ & alive & 1816 & Cottonwood creek & 508590 & 4710790 & 1660 \\
\hline Sceloporus graciosus & $05 / 28 / 96$ & alive & 1830 & Cottonwood creek & 508491 & 4709640 & 1677 \\
\hline Sceloporus graciosus & $05 / 28 / 96$ & alive & 1842 & Cottonwood creek & 508433 & 4709209 & 1702 \\
\hline Sceloporus graciosus & $05 / 29 / 96$ & alive & 1623 & Trib to Cottonwood & 506321 & 4709233 & 1580 \\
\hline Sceloporus graciosus & $05 / 30 / 96$ & alive & 1500 & Juniper $m t n$. & 509523 & 4704993 & 1916 \\
\hline Sceloporus graciosus & $06 / 03 / 96$ & alive & 1146 & Nickel ck. & 517921 & 4711274 & 1632 \\
\hline Sceloporus graciosus & $06 / 03 / 96$ & alive & 1156 & Nickel ck. & 518110 & 4711079 & 1554 \\
\hline Sceloporus graciosus & $06 / 03 / 96$ & alive & 1700 & Trib to Pleasant Valley ck. & & & \\
\hline Sceloporus graciosus & $06 / 03 / 96$ & alive & 1714 & M.F.R. & & & \\
\hline Sceloporus graciosus & $06 / 04 / 96$ & alive & 1601 & Trout ck. & 506935 & 4758178 & 1681 \\
\hline Sceloporus graciosus & $06 / 17 / 96$ & alive & 1906 & Corral Creek & 512551 & 4723493 & 1880 \\
\hline Sceloporus graciosus & $06 / 17 / 96$ & alive & 1954 & Corral Creek & 512551 & 4723493 & 1868 \\
\hline Sceloporus graciosus & $06 / 18 / 96$ & alive & 1632 & Noon Creek & 512106 & 4719386 & 1814 \\
\hline Sceloporus graciosus & $06 / 18 / 96$ & alive & 1645 & Noon Creek & 512321 & 4719669 & 1924 \\
\hline Sceloporus graciosus & $06 / 25 / 96$ & alive & 1401 & Pig Creek & & & \\
\hline Sceloporus occidentalis & $05 / 30 / 96$ & alive & 1500 & Juniper $m t n$. & 509523 & 4704993 & 1916 \\
\hline Sceloporus occidentalis & $05 / 30 / 96$ & alive & 1500 & Juniper $\mathrm{mtn}$. & 509523 & 4704993 & 1916 \\
\hline Sceloporus occidentalis & $06 / 05 / 96$ & alive & 1430 & Indian Meadows rd. & 506415 & 4749839 & 1502 \\
\hline Sceloporus occidentalis & $06 / 05 / 96$ & alive & 1454 & Indian Meadows rd. & 506338 & 4749862 & 1540 \\
\hline Sceloporus occidentalis & $07 / 18 / 96$ & alive & 1458 & Mcbride ck. & 504165 & 4790022 & 1436 \\
\hline Sceloporus occidentalis & $07 / 18 / 96$ & alive & 1623 & Squaw ck. on bank & 509232 & 4804193 & 973 \\
\hline Sceloporus occidentalis & $06 / 10 / 96$ & alive & 928 & Fossil Butte & 545114 & 4773031 & 1000 \\
\hline Sceloporus occidentalis & $06 / 11 / 96$ & alive & 1117 & Mountain Creek & 513548 & 4741246 & 1802 \\
\hline Sceloporus occidentalis & $06 / 11 / 96$ & alive & 1157 & Mountain Creek & 512941 & 4740717 & 1547 \\
\hline Sceloporus occidentalis & $06 / 12 / 96$ & alive & 1145 & Old Man Creek & 510420 & 4743487 & 1589 \\
\hline Sceloporus occidentalis & $06 / 17 / 96$ & alive & 1919 & Corral Creek & 512551 & 4723493 & 1870 \\
\hline Sceloporus occidentalis & $06 / 17 / 96$ & alive & 1919 & Corral Creek & 512551 & 4723493 & 1870 \\
\hline Sceloporus occidentalis & $06 / 17 / 96$ & alive & 1919 & Corral Creek & 512551 & 4723493 & 1870 \\
\hline Sceloporus occidentalis & $06 / 18 / 96$ & alive & 1532 & Noon Creek & 512912 & 4719635 & 1714 \\
\hline
\end{tabular}





\begin{tabular}{|c|c|c|c|c|c|c|c|}
\hline Sceloporus occidentalis & $06 / 18 / 96$ & alive & 1543 & Noon Creek & 512139 & 4719314 & 1783 \\
\hline Sceloporus occidentalis & $06 / 18 / 96$ & dead & 1602 & Noon Creok & 512139 & 4719314 & 1780 \\
\hline Sceloporus occidentalis & $07 / 23 / 96$ & alive & 1556 & on logrbank Jump Cr. & & & \\
\hline Sceloporus occidentalis & $07 / 23 / 96$ & alive & 1630 & on bank Jump Cr. falls. & & & \\
\hline Sceloporus occidentalis & $07 / 23 / 96$ & alive & 1701 & on rock, Jump Cr. falls. & & & \\
\hline Sceloporus occidentalis & $07 / 25 / 96$ & alive & 1224 & Hardtrigger $\mathrm{Cr}$. on bank & 516294 & 4800902 & 1147 \\
\hline Sceloporus occidentalis & $07 / 30 / 96$ & alive & 1848 & Deep Cr. on bank. & 528022 & 4694565 & 1568 \\
\hline Thamnophis elegans & $05 / 28 / 96$ & alive & 1004 & Cottonwood, spring & 508560 & 4708247 & 1745 \\
\hline Thamnophis elegans & $05 / 28 / 96$ & alive & 1158 & Cottonwood creek & 508744 & 4709149 & 1653 \\
\hline Thamnophis elegans & $05 / 28 / 96$ & alive & 1246 & Trib to Cottonwood & 507977 & 4709247 & 1721 \\
\hline Thamnophis elegans & $05 / 28 / 96$ & alive & 1400 & Cottonwood creek & 508262 & 4709431 & 1691 \\
\hline Thamnophis elegans & $05 / 28 / 96$ & alive & 1725 & Cottonwood creek & 508590 & 4710252 & 1760 \\
\hline Thamnophis elegans & $05 / 29 / 96$ & alive & 1242 & Cottonwood creek & 508344 & 4707479 & 1880 \\
\hline Thamnophis elegans & $05 / 29 / 96$ & alive & 1557 & Trib to Cottonwood & 505993 & 4708899 & 1588 \\
\hline Thamnophis elegans & $05 / 27 / 96$ & alive & 1643 & Trib to Cottonwood & 505868 & 4709659 & est. \\
\hline Thamnophis elegans & $05 / 30 / 96$ & alive & 1430 & Trib to Pleasant Valley ck. & 509734 & 4705959 & 1784 \\
\hline Thamnophis elegans & $06 / 03 / 96$ & alive & 1118 & Nickel ck. & 517056 & 4711866 & 1738 \\
\hline Thamnophis elegans & $06 / 03 / 96$ & alive & 1300 & in pond, $T$. to Pleasant Valley & 514022 & 4711816 & 1737 \\
\hline Thamnophis elegans & $06 / 03 / 96$ & alive & 1307 & in pond $T$. to Pleasant Valley & 513589 & 4711689 & 1737 \\
\hline Thamnophis elegans & $06 / 03 / 96$ & alive & 1400 & in pond, T. to Pleasant Valley & 513589 & 4711441 & 1569 \\
\hline Thamnophis elegans & $06 / 03 / 96$ & alive & 1510 & Pleasant Valley ck. & 511668 & 4712703 & 1687 \\
\hline Thamnophis elegans & $06 / 04 / 96$ & alive & 1429 & Trout ck. & 505300 & 4759092 & 1567 \\
\hline Thamnophis elegans & $06 / 04 / 96$ & alive & 1434 & Trout ck. & 505310 & 4758703 & 1580 \\
\hline Thamnophis elegans & $06 / 04 / 96$ & alive & 1436 & Trout ck. & 505315 & 4758710 & 1561 \\
\hline Thamnophis elegans & $06 / 04 / 96$ & alive & 1446 & Trout ck. & 505455 & 4759114 & 1421 \\
\hline Thamnophis elegans & $06 / 04 / 96$ & alive & 1450 & Trout ck. & 505472 & 4759092 & 1424 \\
\hline Thamnophis elegans & $06 / 04 / 96$ & alive & 1501 & Trout ck. & 505315 & 4759148 & 1594 \\
\hline Thamnophis elegans & $07 / 02 / 96$ & alive & 1806 & Indian Creek in grass & 575277 & 4668294 & 1800 \\
\hline Thamnophis elegans & $07 / 02 / 96$ & alive & 1815 & Indian Creek in grass & 575291 & 4668303 & 1572 \\
\hline Thamnophis elegans & $07 / 04 / 96$ & alive & 908 & Battle Creek in sage & 554209 & 4706059 & 1598 \\
\hline Thamnophis elegans & $07 / 04 / 96$ & alive & 1049 & Big Springs Creek on bank in & 551537 & 4702357 & 1465 \\
\hline Thamnophis elegans & $07 / 04 / 96$ & alive & 1109 & Big Springs Creek on bank in & 551495 & 4701939 & 1738 \\
\hline Thamnophis elegans & $07 / 04 / 96$ & alive & 1111 & Big Springs Creek on bank in & 551492 & 4701839 & 1689 \\
\hline Thamnophis elegans & $07 / 04 / 96$ & alive & 1115 & Big Springs Creek on bank in & 551503 & 4701775 & 1777 \\
\hline Thamnophis elegans & $07 / 04 / 96$ & alive & 1117 & Big Springs Creek on bank in & 551498 & 4702028 & 1645 \\
\hline Thamnophis elegans & $07 / 04 / 96$ & alive & 1148 & Big Springs Creek on bank in & 551904 & 4701203 & 1717 \\
\hline Thamnophis elegans & $07 / 04 / 96$ & alive & 1148 & Big Springs Creek on bank in & 551904 & 4701203 & 1717 \\
\hline Thamnophis elegans & $07 / 04 / 96$ & alive & 1154 & Big Springs Creek on bank in & 552040 & 4701158 & 1722 \\
\hline Thamnophis elegans & $07 / 04 / 96$ & alive & 1229 & Big Springs Creek on bank in & 552675 & 4700266 & 1632 \\
\hline Thamnophis elegans & $07 / 04 / 96$ & alive & 1238 & Big Springs Creek on bank in & 552510 & 4700243 & 1625 \\
\hline Thamnophis elegans & $07 / 04 / 96$ & alive & 1243 & Big Springs Creek on bank in & 553044 & 4699965 & 1725 \\
\hline Thamnophis elegans & $07 / 08 / 96$ & alive & 1550 & Camel Creek & 530279 & 4708353 & 1676 \\
\hline Thamnophis elegans & $07 / 09 / 96$ & alive & 1640 & Camel Creek & 530202 & 4708553 & 1618 \\
\hline Thamnophis elegans & $07 / 09 / 96$ & alive & 1333 & on rock in water of Pole Cree & 537531 & 4714656 & 1531 \\
\hline Thamnophis elegans & $07 / 09 / 96$ & alive & 1350 & on rock in water of Pole Cree & 552094 & 4708816 & 1649 \\
\hline Thamnophis elegans & $07 / 10 / 96$ & alive & 1107 & in pond near battle ck. & 556203 & 4702054 & 1659 \\
\hline Thamnophis elegans & $07 / 10 / 96$ & alive & 1107 & in pond near battle ck. & 556203 & 4702054 & 1659 \\
\hline Thamnophis elegans & $07 / 10 / 96$ & alive & 1155 & in battle ck. & 555661 & 4703037 & 1759 \\
\hline Thamnophis elegans & $07 / 15 / 96$ & alive & 1156 & Johnston res. & 513565 & 4772045 & 2022 \\
\hline Thamnophis elegans & $07 / 15 / 96$ & alive & 1235 & Johnston res. & 513506 & 4771967 & 1995 \\
\hline Thamnophis elegans & $07 / 15 / 96$ & alive & 1600 & Johnston res. & 513528 & 4772161 & 1879 \\
\hline Thamnophis elegans & $07 / 15 / 96$ & alive & 1818 & in $\mathrm{H} 2 \mathrm{O}$, cattle pond & 516587 & 4768182 & 1998 \\
\hline Thamnophis elegans & $07 / 16 / 96$ & alive & 1417 & Trib. to Succor ck. & 516428 & 4777000 & 1820 \\
\hline Thamnophis elegans & $07 / 16 / 96$ & alive & 1459 & Succor ck. in water & 516131 & 4776074 & 1569 \\
\hline Thamnophis elegans & $07 / 16 / 96$ & alive & 1517 & Succor ck. in water & 515943 & 4775592 & 1721 \\
\hline Thamnophis elegans & $07 / 16 / 96$ & alive & 1530 & Succor ck in water & 515820 & 4775441 & 1714 \\
\hline Thamnophis elegans & $07 / 16 / 96$ & alive & 1753 & Succor ck. in water & 514507 & 4777702 & 1544 \\
\hline Thamnophis elegans & $07 / 17 / 96$ & alive & 1507 & Jordan ck. on bank & 516334 & 4763959 & 1613 \\
\hline Thamnophis elegans & $07 / 17 / 96$ & alive & 1528 & Jordan ck. on bank & 516623 & 4764200 & 1712 \\
\hline Thamnophis elegans & $07 / 17 / 96$ & alive & 1535 & Jordan ck. on bank & 516621 & 4764226 & 1632 \\
\hline Thamnophis elegans & $07 / 17 / 96$ & alive & 1537 & Jordan ck. on bank & 516537 & 4764248 & 1645 \\
\hline
\end{tabular}





\begin{tabular}{|c|c|c|c|c|c|c|c|}
\hline Thamnophis elegans & $07 / 17 / 96$ & alive & 1545 & Jordan ck. on bank & 516713 & 4764458 & 1735 \\
\hline Thamnophis elegans & $07 / 17 / 96$ & alive & 1552 & Jordan ck. on bank & 516891 & 4764551 & 1710 \\
\hline Thamnophis elegans & $07 / 17 / 96$ & alive & 1914 & Jordan ck. in water & 512223 & 4763886 & 1690 \\
\hline Thamnophis elegans & $07 / 18 / 96$ & alive & 1434 & Mcbride ck. on rocks. & 504306 & 4789736 & 1338 \\
\hline Thamnophis elegans & $07 / 18 / 96$ & alive & 1635 & Squaw ck. on bank & 509000 & 4804097 & 1087 \\
\hline Thamnophis elegans & $06 / 10 / 96$ & alive & 1531 & Deer Creek pond & 519352 & 4745369 & 1699 \\
\hline Thamnophis elegans & $06 / 10 / 96$ & alive & 1531 & Deer Creek pond & 519352 & 4745369 & 1699 \\
\hline Thamnophis elegans & $06 / 10 / 96$ & alive & 1531 & Deer Creek pond & 519352 & 4745369 & 1699 \\
\hline Thamnophis elegans & $06 / 10 / 96$ & alive & 1531 & Deer Creek pond & 519352 & 4745369 & 1699 \\
\hline Thamnophis elegans & $06 / 12 / 96$ & alive & 1347 & Rail Creek & 508722 & 4741779 & 1531 \\
\hline Thamnophis elegans & $06 / 12 / 96$ & alive & 1401 & Rail Creek & 508802 & 4742101 & 1607 \\
\hline Thamnophis elegans & $06 / 17 / 96$ & alive & 2009 & Bogus Creek & 519226 & 4729804 & 1669 \\
\hline Thamnophis elegans & $06 / 19 / 96$ & alive & 1210 & Corral Creek & 511265 & 4722489 & 1718 \\
\hline Thamnophis elegans & $06 / 19 / 96$ & alive & 1358 & Corral Creek & 512081 & 4724168 & 1753 \\
\hline Thamnophis elegans & $06 / 19 / 96$ & alive & 1833 & Indian Meadows road & 506610 & 4740617 & 1667 \\
\hline Thamnophis elegans & $06 / 25 / 96$ & alive & 1220 & Pig Creek Pond, Hwy. 51 & 577410 & 4675038 & 1823 \\
\hline Thamnophis elegans & $06 / 25 / 96$ & alive & 1220 & Pig Creek Pond, Hwy. 51 & 577410 & 4675038 & 1823 \\
\hline Thamnophis elegans & $06 / 25 / 96$ & alive & 1100 & Litte Blue Creek Res. & 573719 & 4683440 & 1494 \\
\hline Thamnophis elegans & $06 / 27 / 96$ & alive & 1209 & In sage of Blue Creek & 566310 & 4687265 & 1585 \\
\hline Thamnophis elegans & $07 / 24 / 96$ & alive & 1452 & Reynolds Cr. in water. & 520199 & 4790464 & 1105 \\
\hline Thamnophis elegans & $07 / 29 / 96$ & road kill & 1210 & Mud Flat road & 507594 & 4708591 & 1626 \\
\hline Thamnophis elegans & $07 / 30 / 96$ & road kill & 1039 & Mud Flat road & 575283 & 4751476 & 765 \\
\hline Thamnophis elegans & $07 / 30 / 96$ & alive & 1821 & Deep Cr. on bank. & 528611 & 4694534 & 1252 \\
\hline Thamnophis elegans & $05 / 20 / 96$ & alive & 1355 & Cottonwood creek & & & \\
\hline Thamnophis elegans & $07 / 31 / 96$ & alive & 1212 & Rock Creek in willows. & 537948 & 4728368 & 1727 \\
\hline Thamnophis elegans & $07 / 31 / 96$ & alive & 1310 & Rock Creek in willows. & & & \\
\hline Thamnophis elegans & $07 / 31 / 96$ & alive & 1320 & Rock Creek in willows. & 538376 & 4727837 & 1549 \\
\hline Thamnophis elegans & $07 / 31 / 96$ & alive & 1321 & Rock Creek in willows. & 538403 & 4727738 & 1727 \\
\hline Thamnophis elegans & $07 / 31 / 96$ & alive & 1338 & Rock Creek in willows. & 538497 & 4727900 & 1769 \\
\hline Thamnophis elegans & $07 / 31 / 96$ & alive & 1347 & Rock Creek in willows. & 538514 & 4727606 & 1645 \\
\hline Thamnophis elegans & $07 / 31 / 96$ & alive & 1357 & Rock Creek in willows. & 538506 & 4727483 & 1631 \\
\hline Thamnophis elegans & $07 / 31 / 96$ & alive & & Rock Creek in willows. & 538352 & 4727165 & 1703 \\
\hline Thamnophis elegans & $07 / 31 / 96$ & alive & & Rock Creek in willows. & & & \\
\hline Thamnophis elegans & $08 / 01 / 96$ & alive & 957 & Stoneman Creek. & 520766 & 4712785 & 1587 \\
\hline Thamnophis elegans & $08 / 01 / 96$ & alive & 957 & Stoneman Creek. & 520766 & 4712785 & 1587 \\
\hline \multirow[t]{2}{*}{ Thamnophis elegans } & $08 / 01 / 96$ & alive & 1007 & Stoneman Creek. & 520776 & 4712860 & 1552 \\
\hline & & & & & 520917 & 4713093 & 1659 \\
\hline Thamnophis elegans & $08 / 01 / 96$ & alive & 1040 & Stoneman Creek. & 520957 & 4713030 & 1542 \\
\hline Thamnophis elegans & $08 / 01 / 96$ & alive & 1120 & Stoneman Creek. & & & \\
\hline Thamnophis elegans & $08 / 01 / 96$ & alive & 1120 & Stoneman Creek. & & & \\
\hline Thamnophis elegans & $08 / 01 / 96$ & alive & 1140 & Stoneman Creek. & & & \\
\hline Thamnophis elegans & $08 / 01 / 96$ & alive & 1202 & Stoneman Creek. & & & \\
\hline Thamnophis elegans & $08 / 18 / 96$ & alive & 1205 & Stoneman Creek. & & & \\
\hline
\end{tabular}




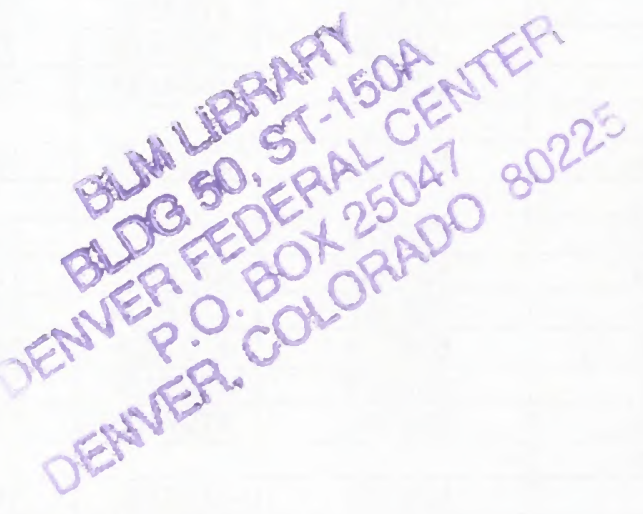




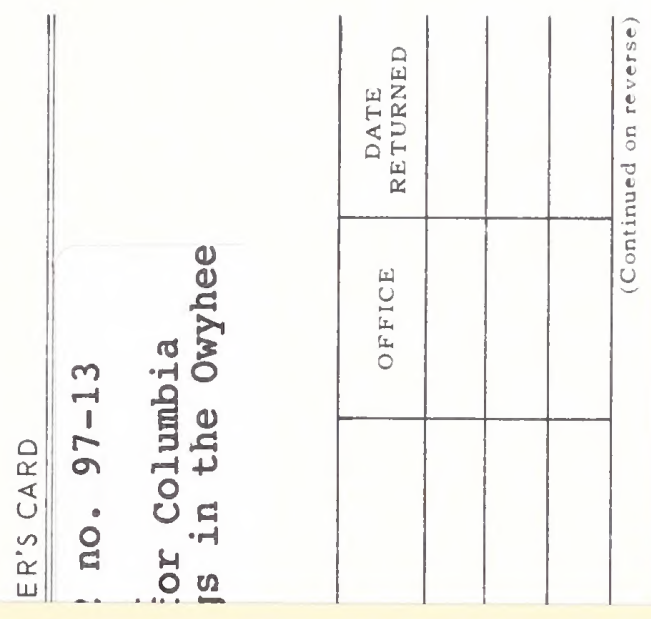

QL 84.2. L352 no. 97-13

88055391

1996 survey for Columbia

spotted frogs in the Owyhee

BLDG 50, ST-150A

DENVER FEDERAL CENTER

P.O. BOX 25047

DENVER, COLORADO 80225 


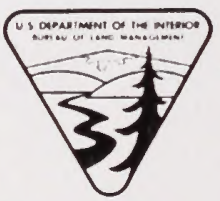

Bureau of Land Management

Idaho State Office

1387 S. Vinnell Way

Boise, Idaho 83709

BLM/ID/PT-97/016+1150 